\title{
Manipulation of RNA Polymerase III by Herpes Simplex Virus-1
}

2

13 * Corresponding author:

14 Neal A. DeLuca

15 Department of Microbiology and Molecular Genetics

16 University of Pittsburgh School of Medicine

$17 \quad 514$ Bridgeside Point II

18450 Technology Dr.

19 Pittsburgh, PA 15219

20 E-mail: ndeluca@pitt.edu

21 Phone:(412) 648-9947

22 FAX: (421) 624-1401

Neal A. DeLuca ${ }^{1 *}$ of Medicine, PA, USA USA Institute, National Institutes of Health, MD, USA 5. Howard Hughes Medical Institute, CA, USA 


\section{ABSTRACT}

RNA Polymerase III (Pol III) transcribes noncoding RNA, including transfer RNA

25 (tRNA), and acts as a pathogen sensor during the innate immune response. To promote

26 enhanced proliferation, the Pol III machinery is commonly targeted during cancer and

27 viral infection. Herein we employ DM-RNA-Seq, 4SU-Seq, ChIP-Seq, and ATAC-Seq to

28 characterize how Herpes Simplex Virus-1 (HSV-1) perturbs the Pol III landscape. We

29 find that HSV-1 stimulates tRNA expression 10-fold, with mature tRNAs exhibiting a 2-

30 fold increase within 12 hours of infection. Perturbation of host tRNA synthesis requires

31 nuclear viral entry, but not synthesis of specific viral transcripts, nascent viral genomes,

32 or viral progeny. Host tRNA with a specific codon bias were not targeted-rather

33 increased transcription was observed from euchromatic, actively transcribed loci. tRNA

34 upregulation is linked to unique crosstalk between the Pol II and III transcriptional

35 machinery. While viral infection is known to mediate host transcriptional shut off and

36 lead to a depletion of Pol II on host mRNA promoters, we find that Pol II binding to tRNA

37 loci increases. Finally, we report Pol III and associated factors bind the HSV genome,

38 which suggests a previously unrecognized role in HSV-1 gene expression. These data

39 provide insight into novel mechanisms by which HSV-1 alters the host nuclear

40 environment, shifting key processes in favor of the pathogen. 


\section{INTRODUCTION}

Herpes Simplex Virus-1 (HSV-1) is a ubiquitous human pathogen which most commonly causes recurrent lesions of the oral and genital mucosa. The virus is associated with a wide range of additional pathologies-including herpes keratitis, herpetic whitlow, and encephalitis, to name a few-representative of the large range of cells permissive to replication. Similar to other herpesviruses, HSV-1 establishes a latent reservoir in the peripheral nervous system and reactivates to cause disease in response to various physiological stimuli.

HSV-1 replicates and assembles almost entirely within the host nucleus, reprogramming the host transcriptional machinery to prioritize expression of $\sim 90$ viral messenger RNAs (mRNAs) (1). These genes are transcribed in a temporally coordinated sequence, such that their protein products are expressed at the appropriate

53 time in the life cycle of the virus $(2,3)$. Immediate early (IE or $\alpha$ ) gene products enable

54 the efficient expression of early $(E$ or $\beta)$ and late $(L$ or $\gamma)$ genes. The protein products of

55 E genes are mostly involved in DNA replication. DNA replication and IE proteins enable

56 the efficient transcription of $L$ genes, which encode the structural components of the

57 virus. DNA replication licenses $L$ promoters, enabling the binding of core Pol II

58 transcription factors, thus activating the initiation of $\mathrm{L}$ transcription (4). Productive HSV-1

59 infection is incredibly rapid, causing a single infected cell to produce progeny between 4

60 and 6 hours $(\mathrm{h})$ postinfection, culminating in $\sim 1,000$ infectious progeny within $18 \mathrm{~h}$.

61 Considering the rapid HSV-1 life cycle, it is perhaps unsurprising that within $6 \mathrm{~h}$ of

62 infection, viral transcripts rise to $50 \%$ of the total mRNA within a host cell. The

63 corresponding decrease in host transcripts is facilitated by two mechanisms i. VHS- 
64 mediated mRNA decay (5-7) and ii. ICP4-DNA mediated decrease of Pol II on mRNA

65 promoters (8-11).

Transcriptional studies of HSV center on mRNA and RNA Polymerase II (Pol II),

67 and thus ignore the potential contributions of other DNA-dependent RNA Polymerases,

68 namely Pol I and Pol III (12, 13). Pol I transcribes a single multi-copy transcript, 45S,

69 which is spliced and processed to produce 5.8S, 18S, and $28 \mathrm{~S}$ ribosomal RNA (rRNA).

70 Pol III transcribes noncoding RNAs, including 5S rRNA, transfer RNA (tRNA), Alu

71 elements, 7SL, 7SK, U6, and select microRNA (miRNA). Pol III also transcribes

72 noncoding RNAs (ncRNA) for various DNA viruses, including: VAl and VAll RNAs of

73 adenovirus (14), EBER1 and EBER2 of Epstein Barr Virus (15) and the tRNA-miRNA

74 encoding RNAs (TMERs) of MHV68 (16-18).

In this study, we explore HSV-1 alteration of the host Pol III transcription

76 landscape. Pol III transcription requires its own unique set of general transcription

77 factors, and the basal promoter requirements have been classified into three different

78 promoter types (19). Type I promoters include 5S rRNA genes, and use the TFIIIB

79 complex (BDP1, TBP, BRF1), TFIIIC complex (GTF3C1, GTF3C2, GTF3C3, GTF3C4,

80 GTF3C5, GTF3C6), and TFIIIA (GTF3A). Type II promoters include tRNAs and require

81 the TFIIIB and TFIIIC complexes. Type III promoters include tRNA-Sec, U6 and 7SK

82 and use distal enhancers including STAF, OCT1, SNAP as well as a distinct TFIIIB

83 complex (BDP1, TBP, BRF2). Each of these promoters consists of distinct combinations

84 of internal cis-acting sites (A, B, C, IE). To add another layer of complexity, recent

85 studies have demonstrated crosstalk between Pol II and Pol III. Highly expressed Pol III

86 transcripts are located in regions of open chromatin adjacent to Pol II promoters (20). 
87 Additionally, Pol II binding is observed at highly expressed Pol III promoters (21), and

88 Pol III occupancy frequently scales with nearby levels of Pol II $(19,22)$.

Viruses have evolved unique mechanisms to invade hosts, alter cellular

90 pathways, and redirect host factors for viral processes. A number of DNA viruses,

91 including adenovirus (23-25), SV40 (26), and Epstein Barr Virus (27), have been shown

92 to increase Pol III transcription in infected cells. These viruses employ a viral regulatory

93 protein (E1A, E1B, T-antigen, and EBNA1) to mediate increase of Pol III transcription

94 factor abundance. Additionally, infection with the gammaherpesvirus MHV68 has been

95 linked to increased expression of murine B2 SINE retrotransposons and pre-tRNAs,

96 suggesting that viral manipulation of Pol III may impact both viral and host Pol III

97 transcription $(28,29)$. HSV-1 has previously been shown to induce the Pol III type II

98 transcript, Alu repeat units $(30,31)$. How HSV-1 affects other Pol III-dependent

99 transcripts and the mechanism behind Alu upregulation is yet unknown.

100

Herein we report a comprehensive characterization of how HSV-1 alters the Pol

101 III transcriptional landscape, ultimately increasing the pool of tRNA available during

102 productive infection. Changes in tRNA levels coincided with increased Pol II recruitment

103 at these loci. This discovery is at odds with the general environment of Pol II depletion

104 from host mRNA promoters which contributes to host transcriptional shut off (8-11). We

105 also report, for the first time, recruitment of Pol III to the HSV-1 genome. Whether this

106 binding event results in production of a Pol III-dependent transcript is still unknown.

107 However, these results suggest a novel role of Pol III in promoting and regulating HSV-1

108 transcription. 


\section{RESULTS}

\section{Impact of HSV-1 Productive Infection on Pol I and III Transcripts}

We began by assessing changes in noncoding RNA species, particularly those expressed by Pol I and III. Human diploid fibroblast (MRC5) cells were mock-infected,

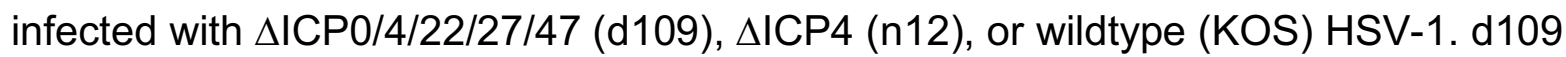
infection lacks synthesis of all viral proteins and viral genomes; however, it robustly stimulates a cGAS-mediated innate immune response $(32,33) . \mathrm{n} 12$ infection is deficient in the synthesis of early $(E)$ and late $(L)$ viral proteins, nascent viral genomes, and viral progeny (34). We observed little change in Pol I transcripts 18S rRNA, 45S pre-rRNA, or 5.8S rRNA using RT-qPCR (Fig. 1A) and northern blot analysis (Fig. 1B). Pol III type I, II, and III transcripts were affected disparately (Fig. 1A-B). Type I transcript 5S rRNA was unaltered by infection, while type II transcripts were strongly upregulated. Infection with n12 and wildtype HSV-1 increased the levels of type II transcripts from 2 to 16 -fold (Fig. 1A-C). This upregulation was most drastic for pre-tRNA, and specific to tRNA with type II promoters as we did not observe upregulation for a tRNA encoded by a type III promoter, namely tRNA selenocysteine (tRNA Sec). We observed an increase-albeit modest $\sim 1.5$-fold-in mature tRNA (Fig. 1C). Type III transcripts U6 snRNA, tRNA-sec, and 7SK decreased minimally during infection (Fig. 1A-B). n12 infection phenocopied transcriptional changes in wild-type infection, indicating that synthesis of $E$ and $L$ viral proteins, nascent viral genomes, or viral progeny were not required for the virus to alter the Pol III transcriptional landscape. In contrast, d109 infection had no impact on the transcriptional landscape, indicating viral nuclear entry or the induction of an innate 
131 immune response is not sufficient to induce tRNA upregulation. These results

132 demonstrate HSV-1 selectively targets and upregulates Pol III type II transcripts.

134 Alteration of the tRNA landscape by HSV-1 Productive Infection

135 To investigate global changes in tRNA species during HSV-1 productive infection, 136 we performed DM-tRNA-Seq (35). Cells were mock-infected or infected with n12 or

137 KOS for 12 hours. Using this technique, we can accurately discriminate between pre138 and mature-tRNA species and between the approximately 500 different tRNA-encoding 139 loci within the host genome. We further delineated tRNA expression by those encoded 140 from the nuclear or mitochondrial (MT) host genome.

141 We found that the total amount of nuclear-encoded tRNA increased 2-fold after 142 infection with wildtype or $\triangle \mathrm{ICP} 4 \mathrm{HSV}-1$ (n12). Pre-tRNA species were more affected 143 than mature-tRNA, increasing 4 and 1.5-fold, respectively (Fig 2A). Nuclear-encoded 144 tRNA expression was altered similarly between KOS and n12 infection (Fig. 2A-C), 145 indicating only early viral life cycle events were required for the phenotype. In contrast, 146 MT-encoded tRNA decreased 4-fold only in wildtype HSV-1 infection (Fig. 2A). We

147 expected this decrease as HSV-1 degrades the host mitochondrial genome during 148 productive infection (36). MT-tRNA changed minimally in n12 infection likely due to the 149 absence of UL12 expression, which is the viral endonuclease responsible for MT150 genome degradation.

151 Since pre-tRNAs were more upregulated than the mature form we hypothesized that 152 infection increased nascent transcription of nuclear-encoded tRNA. To assess this, we 153 pulsed in 4-thiouridine (4SU) for 15 minutes at various stages of infection before 
154 isolating RNA and performing 4SU-Seq. tRNA are very stable ncRNAs, with half-lives of

155 about 3 days. Using 4SU-Seq we were able to assess how nascent transcription shifts,

156 without being confounded by tRNA made prior to infection. Echoing our DM-tRNA-Seq

157 results, nuclear-encoded tRNA increased and MT-encoded tRNA decreased after

158 infection (Fig. 2D). Nascent nuclear tRNA levels were increased 10-fold at 12 hpi (Fig.

159 2D). In Fig. 2E we show data for the top differentially expressed (DE) mature ( $p$-value

$160<0.05, \log _{2}$ fold change KOS/uninfected $>0.5$ ) and pre- ( $p$-value $<0.05, \log _{2}$ fold change

161 KOS/uninfected >2) tRNA species in the DM-tRNA-Seq dataset (Supplemental Table 1).

162 We observed an increase in nascent tRNA detected for these DE mature- and pre-

163 tRNAs (Fig. 2E).

164 We next assessed whether there was enrichment for select isodecoders among the

165 tRNA species altered by infection (Fig. S2-1). The HSV-1 genome is unusually GC-rich,

$166 \sim 68 \%$, which means the viral coding sequence relies more heavily on tRNA with GC-

167 rich anticodons (Fig. S2-1B). Our breakdown of DE tRNA found that the isodecoders

168 altered did not target only GC-rich species (Fig. S2-1). Generally, altered mature and

169 pre-tRNA loci isodecoders appeared random (Fig. S2-1C). Additionally, we did not

170 observe a shift in the identity of tRNA expressed, as silenced tRNA loci were not

171 suddenly expressed or vice versa (Fig. S2-1C). Further study of the actual codon usage

172 truly sampled during infection would need to be determined, this was merely a

173 theoretical codon usage assuming each HSV-1 protein was synthesized in the same

174 amount. Additional work is necessary to determine which, if any, of the differentially

175 expressed tRNAs are rate limiting in viral translation. 
Thus far we have focused on upregulated tRNA, however there was a smaller subset of tRNA ( $n=32$ pre-tRNA, $n=10$ mature tRNA) downregulated by HSV-1 infection

178 (Fig. 2B-C). We evaluated the genomic position of up- and down-regulated tRNA and

179 found that downregulated tRNA loci were located in close proximity to Pol II gene

180 promoters (Fig S2-2). $\sim 50 \%$ of downregulated tRNA loci were located within $3 \mathrm{kbp}$ of a

181 protein-coding gene promoter, whereas only $\sim 15 \%$ of upregulated tRNA loci or all tRNA

182 loci were promoter-adjacent (Fig S2-2D). These data suggest downregulated tRNA

183 targets may be linked to the absence of host transcription at adjacent mRNA promoters.

184 Based on these results it is unlikely that tRNA upregulation is due to Pol II-

185 transcriptional run off or -transcriptional interference.

Defining HSV-1 processes required for tRNA upregulation

Our results in Fig. 1 limit the viral processes which may be critical for tRNA upregulation, including: i. immediate early (IE) proteins other than ICP4, ii. viral ncRNA,

190 or iii. amount, but not identity, of viral transcripts. To discriminate between these

191 hypotheses, we infected MRC5 cells with various HSV-1 mutants and assessed tRNA

192 abundance by Northern Blot (Fig. 3). Mutants for the viral IE proteins-n12 (ICP4),

193 5d11.2 (ICP27), n199 (ICP22), n212 (ICP0)—induced pre-tRNA-Ile indicating that these

194 proteins are not themselves responsible for tRNA upregulation (Fig. 3A-B). Surprisingly, 195 infection with hp66, a mutant defective for genome replication, did not induce host tRNA 196 (Fig. 3A-B). This result was phenocopied in cells treated with specific inhibitors of HSV1971 genome replication (phosphonoacetic acid and acyclovir) and infected with wildtype 198 HSV-1 (Supplemental Fig. 3-1C). Since other mutants in the panel were also defective 
199 for viral genome replication, this life stage could not be responsible for induction of host

200 tRNA (Fig. 3C). Results for other mutants in the panel determined that synthesis of viral

201 L proteins, genome replication and virion assembly were not processes required for

202 induction (Fig. 1, 3A-C).

We performed Ribominus-RNA-Seq on these samples to assess differentially

204 expressed viral genes (Fig. 3D-F). We employed ERCC spike-in controls to normalize expression relative to rRNA—which remain steady during HSV-1 productive infectionallowing a quantitative comparison of samples with varying levels of host shut off. We

207 quantified differentially expressed viral genes, focusing on viral transcripts upregulated 208 relative to hp66 infection (Fig. 3D). This identified ICP0, ICP47, ICP4, RL1, and LAT as 209 possible candidates responsible for the differential tRNA phenotype in hp66. These

210 regions of the genome also contain viral ncRNA transcripts: Latency-Associated

211 Transcript (LAT), miRNAs, and L/STs. We used genomic lesion mutants (d120, d92,

212 d99, R3616 F- $\Delta$ ICP47), rather than nonsense mutants, to clarify our findings. Consistent

213 with Fig. 3A-B, we found that the coding regions of ICPO and ICP4 were not required for

214 tRNA upregulation (Supplemental Fig. 3-1). Additionally, we found mutants with lesions

215 for ICP47, RL1 or LAT induced host tRNA species (Supplemental Fig. 3-1B). Taken

216 together we concluded that ICP0, ICP47, ICP4, RL1, LAT, and miRNAs encoded within

217 LAT or ICP4 were not responsible for tRNA upregulation.

218 This led us to our final hypothesis, in which the amount of viral transcripts-but

219 not the identity of those expressed-is critical for tRNA upregulation. As expected, we

220 observed robust viral transcription in n212 and wild-type KOS infection (Fig. 3E-F).

221 ICP4, ICP27, and ICP22 all function to promote viral transcript accumulation, thus 
222 mutants defective for these proteins had significantly reduced viral transcript levels, $\sim 7-$

223 fold compared to wildtype (Fig. 3D-E). Supporting our hypothesis, hp66 was the most

224 defective for total viral transcription, 20-fold compared to wildtype (Fig. 3E-F). These

225 data taken together conclude that tRNA upregulation requires nuclear viral entry, but not

226 synthesis of specific viral transcripts, nascent viral genomes, or viral progeny. These

227 findings support a link between the amount, but not identity, of viral transcripts and

228 tRNA upregulation.

230 Changes to Pol III GTF binding after HSV-1 infection.

231 Next, we tested if infection altered recruitment of the Pol III transcription machinery

232 to tRNA loci. We also assessed recruitment of the catalytic subunit of Pol II, POLR2A.

233 We performed ChIP-Seq in MRC5 cells mock-infected or infected with KOS for 2, 4, or 6

234 hours. Of the transcription machinery tested-POLR3A, BRF1, GTF3C5, TBP,

235 POLR2A — we found that POLR2A had increased binding, by 2-fold, to tRNA loci (Fig.

236 4A-C). Binding of TBP to tRNA loci also showed a moderate increase, but not as strong

237 as POLR2A. Concurrent with prior findings, POLR2A binding to mRNA genes

238 decreased drastically during infection, a phenomenon that promotes host shut off (8-11)

239 (Fig. 4A). tRNA loci with increased POLR2A recruitment were found in accessible

240 regions of the genome (Fig. 4B) and DM-tRNA-Seq results for these loci found

241 increased accumulation after infection (Fig. 4B-C). We did not observe increased

242 recruitment of the catalytic subunit of Pol III, POLR3A, despite our earlier finding that

243 tRNA loci had increased transcriptional output (Fig. 2D-E). The absence of increased

244 Pol III promoter recruitment indicates initiation rates were not solely altered. This 
245 suggests that the increased rate of tRNA transcription is caused by a simultaneous

246 increase in Pol III initiation and elongation rates (37). While other studies have found the

247 Pol II and III transcriptional machinery to be linked, it is still unknown exactly how Pol II

248 enhances or regulates Pol III transcription. Further study is required to determine the

249 mechanism by which Pol II enhances tRNA transcription.

250 To determine whether transcriptional host shut off and tRNA upregulation may be

251 linked phenomena, we performed ChIP-Seq for POLR2A on human fibroblasts after

252 infection with d109, n12, 5d11.2, n199, and KOS for 6 hours (Fig. S4-1). We observed

253 increased recruitment of POLR2A to tRNA loci for all viruses tested, with the exception

254 of d109 (Fig. S4-1A-B). Host shut off is dependent on the presence of ICP4, and scales

255 with viral genome copy number $(10,11)$. Consistent with this, we only observed

256 depletion of Pol II from host promoters after infection with 5dl1.2, n199, and KOS

257 infection (Fig. S4-1C-D). The absence of Pol II recruitment to tRNA loci after d109

258 infection agrees with our earlier findings that d109 infection does not cause tRNA

259 upregulation (Fig. 1 and 3).

260 To assess how HSV-1 promotes Pol II recruitment at tRNA loci we investigated

261 changes in transcription factor expression and availability. We performed polyA-

262 selected RNA-Seq on human fibroblasts mock-infected or infected with KOS for 2, 4, 6,

2638 and $12 \mathrm{~h}$ (Fig. S4-2). In line with the global reduction of host mRNA species following

264 HSV-1 infection (Fig. S4-2A), by 12 hpi transcripts for most of the Pol II and III

265 machinery had decreased between 2 to 32-fold (Fig. S4-2B-C). The one exception was

266 POLR2A, for which we observed a slight increase in transcript abundance, peaking

267 around $4 \mathrm{hpi}$ (Fig. S4-2B). We next assessed whether these transcriptional changes 
268 resulted in altered protein expression (Fig. S4-3). While POLR2A transcript abundance

269 increased around $4 \mathrm{hpi}$, its protein expression levels decreased $\sim 4$-fold by 12 hpi (Fig.

270 S4-3B. Again, we observed a marked decrease in protein expression levels for all

271 transcription machinery assessed, with the exception of POU2F1 and SP1. These

272 cellular enhancers are of particular note because they promote Pol III Type III

273 transcription and are also critical for VP16-mediated enhancement of viral immediate

274 early transcription (38-41). Since these enhancers are not required for Pol III type II

275 transcription, we find it unlikely they contribute to tRNA upregulation, however further

276 work is required. Ultimately these results do not explain enhanced Pol II recruitment to

277 tRNA in an environment where their transcription and protein expression levels are

278 globally decreased.

279 Another mechanism by which HSV-1 alters the host environment is by remodeling

280 the nucleus into sub-domains. HSV-1 assembles within replication compartments

281 enriched for utilized host factors, such as transcription and replication machinery, while

282 excluding negative host factors, most notably histones (42). We imaged Vero cells that

283 were uninfected (Fig. S4-4A), infected pre-replication (Fig. S4-4B), and infected post-

284 replication (Fig. S4-4C-D). We used the nucleoside analog, EdC, to specifically label

285 viral genomes and then stained for various components of the transcriptional machinery

286 including: POLR2A, POLR3A, POLR3G, BRF1, and GTF3C5. We observed a strong

287 reorganization to viral replication compartments for all host factors tested. Of note, only

288 POLR2A colocalized with viral input genomes (Fig. S4-4B). All other components

289 colocalized with the viral genome only after formation of viral replication compartments

290 and multiple rounds of viral genome replication had occurred (Fig. S4-4D). Based on 
291 these results, it is unlikely that HSV-1 promotes Pol II recruitment to tRNA loci by

292 enhancing the local concentration gradient. Additionally, most factors reorganized to

293 viral replication compartments (Fig. S4-4), suggesting decreased recruitment to the host

294 genome-an observation in line with our ChIP-Seq results showing global decrease of

295 POLR2A and TBP from host mRNA promoters and slight decrease of POL3A, BRF1,

296 and GTF3C5 from tRNA loci (Fig. 4A). These results mirror the global environment of

297 host transcriptional repression present in HSV-1 infection, wherein tRNA appear to be

298 the rare outlier.

\section{Recruitment of Pol III to the HSV-1 genome}

301 Classically all HSV-1 transcripts are defined as Pol II-dependent (43). However, 302 there are examples of other DNA viruses that encode Pol III transcripts; the VAI and 303 VAll RNAs of adenovirus (14), EBER1 and EBER2 of Epstein Barr Virus (15), and the 304 tRNA-micro RNA (miRNA) encoding RNAs (TMERs) of MHV68 (16-18). We examined 305 our ChIP-Seq data for binding of the Pol III machinery to the HSV-1 genome. We 306 observed strong, distinct recruitment of the catalytic subunit of Pol III, POLR3A, to the 307 viral genome (Fig. 5A). Of the transcription factors tested-POLR3A, POLR2A, TBP, 308 GTF3A, GTF3C5, BRF1, BRF2, ICP4-POLR3A binding most closely resembled 309 POLR2A (Fig. 5B). POLR3A binding to the viral genome was most prominent during 310 early viral infection (pre-replication), and occurred independent of the major viral 311 transcriptional activator, ICP4 (Fig. S5-1 and S5-2). There were a few prominent 312 instances where POLR3A binding did not mimic POLR2A, namely within the latency 
313 associated transcript (LAT) intron, at the promoter of RL1, and intergenic between US9

314 and US10.

\section{Unique GTF context of viral Pol III binding}

317 A closer analysis of the Pol III machinery recruited to the viral genome revealed two

318 POLR3A binding contexts: 1. Pol II Coincident-with TBP, GTF3C5, GTF3C6, and

319 GTF3A, or 2. Pol II Independent-with GTF3C1 and BRF2 (Fig. 6). These binding

320 contexts are drastically different from those characterized $(19,20)$ and observed in our

321 own data for host promoters (Fig S6-1 and S6-2). Notably the components of the TFIIIC

322 complex, GTF3C1 to GTF3C6, always bind coincident on host Pol III type II promoters.

323 On the HSV-1 genome, binding of GTF3C2 and GTF3C3 was largely absent (Fig. S6-

324 3). GTF3C1, GTF3C5, and GTF3C6 were recruited to the viral genome, however the

325 binding pattern of GTF3C1 did not coincide with the other TFIIIC subunits present (Fig.

326 S6-3). Perhaps the most interesting binding pattern is for the Pol III Type III transcription

327 factor, BRF2. This factor has very select host binding with around a dozen targets (19),

328 an observation consistent with our dataset (Fig. S6-1). BRF2 bound robustly within the

329 LAT intron, coincident with a Pol Il-independent POLR3A binding event (Fig. 6). This

330 binding context requires further investigation to identify what viral transcripts may be 331 produced from this locus. 


\section{DISCUSSION}

Herein we characterized how HSV-1 affects Pol III mediated transcription (Fig.

334 7), a pathway commonly dysregulated by cancer and pathogens. In prior work, HSV-1

335 has been shown to induce the Pol III type II transcript, Alu (30, 31). Echoing these

336 results, we found that HSV-1 targets and upregulates additional Pol III type II

337 transcripts. This includes a 2-fold increase in total abundance of tRNA, and a 10-fold

338 increase in nascent levels of tRNA. We did not observe increased recruitment of Pol III

339 to tRNA. Given these results, we propose that tRNA upregulation is caused by a

340 simultaneous increase in Pol III initiation and elongation rates (37). Given the short half-

341 life of pre-tRNAs, decreased turnover may also contribute to increased nascent

342 abundance. Further work would need to be done to determine whether turnover rates

343 are altered during HSV-1 infection.

$344 \quad$ We identified 15 mature- and 66 pre-tRNA upregulated within 12 hours of HSV-1

345 infection. Exactly which, if any, of these upregulated tRNA species may be rate limiting

346 during viral expression requires further study. There is evidence among other viruses for

347 this hypothesis, for instance HIV lytic replication induces tRNA expression (44, 45). This

348 is so critical that the host innate immune response combats HIV by targeting this

349 pathway via the interferon responsive gene, SLFN11 (46). HSV-1 mediated tRNA

350 upregulation is at odds with the general environment of host transcriptional shut off

351 mediated by the virus, including downregulation of most protein-coding mRNA and Pol

352 III type III transcripts. We posit that HSV-mediated tRNA upregulation is required for

353 robust productive replication, wherein a single infected cell can synthesize up to 30,000

354 viral progeny within a 12-hour window. During this time the virus must produce $\sim 90$ viral 
355 proteins at exponential rates to facilitate genome replication and virion assembly.

356 Alternatively, increased tRNA expression may be critical in infection scenarios where

357 host shut off is less pronounced and there is more competition for translation resources.

358 Another possible option is that an increase in Pol III transcripts may result in an

359 overabundance of RNA molecules that have exposed 5'-triphosphates, which serve as

360 substrates for RNA sensors, such as RIG-I (47). HSV-1 effectively blocks the innate

361 immune response during productive infection-the exact mechanism behind nuclear

362 sensing is still at question (48). HSV-1 perturbation of the Pol III landscape may have a

363 negative side effect in stimulating an innate immune pathway that the virus must quickly

364 block or outstrip in a bid to productively replicate.

365 To determine the mechanism by which HSV-1 induces tRNA transcription, we

366 tested various hypotheses including altered Pol III transcription factor expression,

367 recruitment, or localized concentration. HSV-1 productive infection resulted in a

368 decrease for all three. Decreased Pol III machinery expression suggests that the

369 mechanism by which HSV induces tRNA synthesis differs from other viruses. Namely

370 adenovirus (23-25), SV40 (26), and Epstein Barr Virus (27) which increase the

371 abundance of Pol III transcription factors by viral regulatory proteins E1A, E1B, T-

372 antigen, and EBNA1. We then turned to another possible option, namely crosstalk

373 between the Pol II and III transcription machinery. Recent work has increasingly

374 demonstrated a dependence and interplay between the Pol II and III machinery (19-22,

375 49). In a global environment of Pol II depletion from host genes, we were surprised by a

376 2-fold increase in Pol II recruitment to tRNA loci. Recruitment of Pol II to tRNA genes

377 only occurred upon infection of HSV-1 mutants that also had increased tRNA 
378 abundance. Additionally, recruitment of Pol II to tRNA genes occurred independent of

379 host transcriptional shut down. The question now becomes, how is Pol II enhancing

380 tRNA transcription rates? We propose three potential options: i. Pol II alters the

381 chromatic environment at bound tRNA, ii. enhances the rate of Pol III termination or re-

382 initiation, or iii. functions itself to transcribe tRNA.

383 Infection with a panel of HSV-1 mutants allowed us to closely characterize viral

384 life events critical for tRNA upregulation. We found that nuclear viral entry was required,

385 but synthesis of $E$ and $L$ transcripts, nascent viral genomes, and viral genomes was not

386 required. Furthermore host shut off was not necessary for tRNA upregulation,

387 suggesting a different mechanism of induction than MHV68 perturbation of the tRNA

388 landscape (29). Our results define a narrow window of viral processes which may be

389 critical for tRNA upregulation, namely entry of the viral genome and synthesis of viral

390 transcripts - with no dependence on exactly which transcripts are made. We propose

391 that changes in the pool of tRNA may be caused by a common feature among HSV-1

392 transcripts, including: GC content of $\sim 68 \%$, propensity to form G-quadruplexes, and

393 high density of complementary transcripts. Alternatively, tRNA abundance profiles are

394 known to change drastically in response to various stimuli including oxidative stress,

395 osmotic stress, temperature stress, and diauxic shift (50). Productive viral infection

396 takes an extreme toll on host cell homeostasis, altering levels of metabolites including

397 nucleotides and amino acids (51), inducing an unfolded protein response (52), and

398 dysregulating the DNA damage response (53). Most notably HSV-1 transcripts are

399 composed from a high density of intrinsically disordered domains, and due to the rapid

400 rate of synthesis are prone to misfolding and aggregation-traits that robustly trigger a 
401 UPR. Any one of these cellular feedback loops could be hijacked by HSV-1 to increase

402 the pool of tRNA immediately available for viral use.

403 Thus far, we have focused on how the virus alters host Pol III transcription.

404 However, various viruses including adenovirus, Epstein Barr Virus, and murine

405 herpesvirus-68 possess Pol III-dependent viral transcripts (14-18). These RNAs have

406 functions that combat the innate antiviral response, contribute to latency, and play a role

407 in transformation. Our data suggests that Pol III may play novel roles in HSV-1

408 transcription. We observed Pol III binding to the viral genome in two conformations: i.

409 coincident with RNA Pol II, or ii. coincident with the canonical Pol III type III transcription

410 factor, BRF2. The latter binding event occurred in a region of the genome where

411 latency-derived transcripts originate, suggesting a cell-type specificity and potential role

412 in viral persistence and reactivation. These L/S joint region of the viral genome is rich

413 with ncRNA, including LAT (54), L/STs (55), and microRNAs (56). Pol II promoters have

414 been associated with the genesis of the LAT and L/STs $(57,58)$, and we have also

415 shown that these promoters function in a reconstituted Pol II in vitro transcription

416 systems $(59,60)$. However, these studies do not preclude the possibility that there are

417 alternative mechanisms to transcribe these RNAs, the microRNAs, or other noncoding

418 RNAs yet to be discovered. Herein we provide the first evidence for a putative Pol III

419 transcript derived from the HSV-1 genome. 


\section{ACKNOWLEDGEMENTS:}

421 Thanks to Hannah Fox for datasets and Jill Dembowski for thoughtful

422 discussions. NIH grants R01-Al030612 and R21-Al156065 to N.A. D. NIH grants

423 T32-Al060525 and F31-Al36251 to S. E. D. American Cancer Society Postdoctoral

424 Award 131370-PF-17-245-01-MPC to J.M.T. NIH grant R01-Al147183 to B.A.G. 


\section{FIGURE LEGENDS:}

\section{Fig. 1 Impact of HSV-1 Productive Infection on Pol I and III Transcripts}

428 Human fibroblast cells were mock-infected or infected with d109, n12, or wild-type HSV-

4291 (KOS). RNA was isolated at indicated times, and A) RT-qPCR or B-C) northern blots

430 were used to assess transcript abundance. A) Data is the average of biological triplicate

431 experiments, and values are average $\log _{2}$ fold change of infected over uninfected

432 samples. cDNA copy number was calculated as a function of total RNA used for reverse

433 transcription. B-C) Images are representative northern blots from biological duplicate

434 experiments.

436 Fig. 2 Alteration of the tRNA landscape by HSV-1 Productive Infection

437 Human fibroblasts were mock-infected or infected with $\Delta \mathrm{ICP} 4$ (n12) or wildtype HSV-1

438 (KOS) and RNA was isolated for DM-tRNA-Seq or 4SU-Seq. We used the tRNA

439 assembly for hg38 predicted by GtRNAdb for mapping pre- or mature nuclear and

440 mitochondrial (MT)-encoded tRNA. A, B, C, E) For DM-tRNA-Seq, RNA was collected

$44112 \mathrm{hpi}$ and data was normalized to an internal spike-in control and the size in kb of each

442 tRNA. A) Statistical values were generated from a paired t-test, where * indicates a p-

443 value of $<0.05$, or $* *<0.01$. B-C) Differential expression analysis values are $\log _{2}$ fold

444 change (infected/uninfected) versus the false-discovery rate (FDR) p-value. D-E) 4SU

445 was pulsed in for 15 minutes at the time (hpi) indicated to label nascent RNA. Data was

446 normalized to rRNA mapped reads and the size in kb of each tRNA. E) Heatmaps for

447 upregulated mature- and pre-tRNA. Differentially expressed (DE) mature-tRNA were

448 defined as $p$-value $<0.05, \log _{2}$ fold change (KOS/uninfected) $>0.5$. DE pre-tRNA were 
449 defined as $p$-value $<0.05, \log _{2}$ fold change $(\mathrm{KOS} /$ uninfected $)>2$. Data with a $p$-value

$450>0.05$ was not plotted and is white in the heatmaps.

\section{Supplemental Table 1 DM-tRNA-Seq data}

453 Human fibroblasts were mock-infected or infected with $\Delta \mathrm{ICP} 4$ (n12) or wildtype HSV-1

454 (KOS) for 12 hours. DM-tRNA-Seq was performed, data is the average of four data

455 points consisting of two biological replicate experiments each containing two technical

456 replicates. Data was normalized to an internal spike-in control and the size in kb of each

457 tRNA.

Supplemental Fig. 2-1 Distribution of tRNA isodecoders changed in HSV-1

460 infection.

461 Analysis of isodecoder frequency among DE pre- and mature-tRNA from the DM-tRNA-

462 Seq dataset. "DE-Up" had $\log _{2}$ fold change of KOS/uninfected of $\geq 0.5$ and p-value of <

463 0.05. "DE-Down" had $\log _{2}$ fold change of KOS/uninfected of $\leq-0.5$ and $p$-value of $<$

464 0.05. As tRNA genes are degenerate we summarized by target and anticodon

465 (isodecoder). A) Distribution of affected tRNA isodecoders as a function of DE targets.

466 B) Using the annotated coding sequence of HSV-1 we broke down the theoretical

467 distribution of codon usage, assuming all viral proteins are made equally. Since the

468 HSV-1 genome is $\sim 68 \%$ GC content, isodecoder usage is skewed towards those with

469 GC-rich codons. C) Distribution of affected tRNA isodecoders as a function of those

470 expressed in our experimental system. To classify a tRNA as expressed or "detected" in 
471 our experimental system, we required a cut off of at least 100 normalized mapped reads

472 in uninfected or infected conditions.

474 Supplemental Fig. 2-2 Relative localization of DE tRNA genes to other host genes.

475 Analysis of relative genomic position among DE pre- and mature-tRNA from the DM-

476 tRNA-Seq dataset. "DE-Up" had $\log _{2}$ fold change of KOS/uninfected of $\geq 1$ and $p$-value

477 of $<0.05$. "DE-Down" had Log fold change of KOS/uninfected of $\leq-1$ and $p$-value of $<$

478 0.05. A-B) Relative position was analyzed for DE tRNA to promoters from all annotated

479 genes, excluding tRNA. C-D) Relative position was analyzed for DE tRNA to promoters

480 from only protein-coding genes. A, C) Relative position as a function of all tRNA

481 analyzed. B, D) Percentage of total targets that were located with $3 \mathrm{~kb}$ of a promoter.

483 Fig. 3 Defining HSV-1 processes required for tRNA upregulation.

484 Human fibroblast cells were mock-infected or infected with n12, 5dl1.2, n212, n199, 485 d109, hp66, or wild-type HSV-1 (KOS). RNA was isolated at $12 \mathrm{hpi}$, and A-B) Northern 486 blots, or D-F) Ribo-depleted Total RNA-Seq was used to assess transcript abundance.

487 A) Data is the average of biological replicates, error bars are standard deviation. B)

488 Representative northern blot image of the data quantified in A. C) Summary of

489 phenotypic differences between HSV-1 mutants used. D) Heatmaps for viral transcripts

490 clustered by kinetic class with yellow as immediate early, green as early, blue as leaky

491 late (L1), purple as true late (L2), and black as latency (LAT). Differentially expressed

492 transcripts are highlighted with asterisks. E, F) All RNA-Seq reads mapping to the host 493 or viral genome assembly plotted as abundance (million ERCC spike-in reads per kbp, 
494 Norm MR) or as the ratio of normalized viral transcripts: normalized host transcripts

495 (Viral:Host Ratio).

496

497 Supplemental Fig. 3-1 Refining HSV-1 gene expression required for tRNA 498 upregulation.

499 A, D) Details of strain genotypic and phenotype differences. B) Human fibroblast cells 500 were mock-infected or infected with n12, d120, d92, 5d11.2, n212, d99, n199, R3616, 501 wild-type HSV-1 strain KOS, or wild-type HSV-1 strain F. RNA was isolated at 12 hpi, 502 and Northern blots were used to assess transcript abundance. Representative images

503 from biological duplicates are shown. C) $300 \mathrm{ug} / \mathrm{mL}$ phosphonoacetic acid (PAA) or 100

504 uM acyclovir (ACV) was used to treat mock-infected or KOS-infected cells at 0 h. RNA 505 was collected at $12 \mathrm{~h}$ and assessed by Northern blots. Representative images from 506 biological duplicates are shown.

Fig. 4 Changes to Pol III GTF binding after HSV-1 infection.

509 A-C) Human fibroblasts were mock-infected or infected with wildtype HSV-1 (KOS) and

510 ChIP-Seq $(A)$, ATAC-Seq $(B)$, or DM-tRNA-Seq $(C)$ was performed. ChIP-Seq data is

511 from biological duplicate experiments and normalized for sequencing depth and cellular

512 genome sampling. ATAC-Seq data is from biological duplicate experiments and

513 normalized for sequencing depth, excluding MT reads. DM-tRNA-Seq data is

514 normalized as described in Fig. 2. A) The average $\log _{2}$ fold change for infected over the

515 matched uninfected dataset from $2 \mathrm{~kb}$ upstream of TSS to $2 \mathrm{~kb}$ downstream of TES for

516 all mRNA or tRNA loci. B) Heatmaps for normalized sequencing data mapped from $2 \mathrm{~kb}$ 
517 upstream of TSS to $2 \mathrm{~kb}$ downstream of TES for tRNA loci. C) Traces of representative

518 tRNA genes, y-axes minimum and maximum are shown in brackets.

520 Supplemental Fig. 4-1 Pol II depletion from host mRNA and enrichment on tRNA

521 are not linked.

522 Human fibroblasts were mock-infected or infected with d109 (4ICP0/4/22/27/47), n12

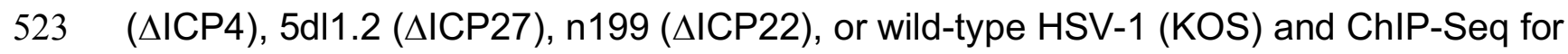

524 POLR2A was performed. ChIP-Seq data is from biological triplicate experiments and

525 normalized for sequencing depth and cellular genome sampling. The average $\log _{2}$ fold

526 change of infected/uninfected signal from $2 \mathrm{~kb}$ upstream of TSS to $2 \mathrm{~kb}$ downstream of

527 TES for all mRNA or tRNA loci. A,C) Data plotted is average of biological triplicates

528 (solid line) with standard deviation (dashed line, shaded). B, D) Data plotted is average

529 of biological triplicates.

531 Supplemental Fig. 4-2 Transcriptomic changes of Pol II and III machinery during

532 HSV-1 productive infection

533 Human fibroblasts were mock-infected or infected with wildtype HSV-1 (KOS) for the

534 indicated times. PolyA-selected RNA-Seq was performed. Data is the average of

535 biological triplicate experiments and error bars are standard deviation. A) Global

536 changes are represented as number of reads mapped to viral or host genome per total

537 reads sequenced (\% Total Reads). B-C) Mapped reads per billion total reads per

538 kilobase pair (MR/BTR/Kb), or $\log _{2}$ fold change of infected over uninfected cells

$539\left(\log _{2} \mathrm{FC}\right)$. 
Supplemental Fig. 4-3 Proteomic changes of Pol II and III machinery during HSV-1

542 productive infection

543 A) Tandem mass tag mass spectrometry (TMT-MS) data from mock- or HSV-1 infected

544 HaCaT cells (human immortalized keratinocytes), published in (61). B) Human fibroblast

545 (MRC5) cells were mock-infected or infected with d109 ( $\Delta \mathrm{IE}$ 's) for 4 hours, n12 $(\Delta \mathrm{ICP} 4)$

546 for 2 hours, or WT (KOS) HSV-1 for 2, 4, 6, 8 or 12 hours. Protein was isolated and

547 measured via LiCor-Western blot for indicated proteins. Each data point is a biological

548 replicate.

550 Supplemental Fig. 4-4 Altered nuclear organization of the Pol III transcription

551 machinery

552 Vero cells were A) mock-infected or B-D) infected with wildtype HSV-1 (KOS) for the

553 indicated time. B) To image input viral genomes we infected with an EdC-prelabed viral

554 stock. C-D) To image nascently replicated viral genomes we pulsed EdC at the

555 indicated time points before fixing. Cells were fixed and EdC labeled DNA was tagged

556 with alexa fluor 488 to visualize viral genomes (green) and cellular proteins were

557 visualized by immunofluorescence (red). Nuclei were labeled with Hoechst (blue).

558 Images are representative of biological duplicate experiments.

560 Fig. 5 Recruitment of Pol III to the HSV-1 genome

561 Human fibroblasts were infected with n12 ( $\mathrm{I}$ CP4) or KOS (WT) HSV-1 for 2 h. ChIP-

562 Seq was performed for POLR3A, POLR2A, TBP, GTF3A, GTF3C5, BRF1, BRF2, and 
563 ICP4. Data is the average of biological duplicates and normalized for sequencing depth

564 and viral genome copy number. A) Traces of POLR2A and POLR3A binding to the

565 unique long (UL), joint, and unique short (US) regions of the HSV-1 genome

566 (KT899744.1 assembly). Y-axes maximum and minimum values are listed within

567 brackets. Viral CDS are listed below, with colors indicating transcriptional class:

568 immediate early (yellow), early (green), leaky late (blue), true late (purple), unclassified

569 (grey). We have also annotated additional genomic features such as TATA boxes (red)

570 and ncRNA (white). Regions where POLR3A bound distinct from POLR2A are

571 highlighted in yellow. B) Principal component analysis of GTF binding profiles for the

572 viral genome, these were calculated by binning (10 bp) normalized traces.

573

574 Supplemental Fig. 5-1 Pol III machinery recruitment to the HSV-1 genome during

575 early infection

576 Human fibroblasts were infected with n12 ( $\triangle \mathrm{ICP} 4)$ or KOS (WT) HSV-1 for 2 h. ChIP-

577 Seq was performed for POLR3A, POLR2A, TBP, GTF3A, GTF3C5, BRF1, and BRF2.

578 Data is the average of biological duplicates and normalized for sequencing depth and

579 viral genome copy number. Traces of binding to the unique long (UL), joint, and unique

580 short (US) regions of the HSV-1 genome (KT899744.1 assembly). Y-axes maximum

581 and minimum values are listed within brackets. Viral CDS are listed below, with colors

582 indicating transcriptional class: immediate early (yellow), early (green), leaky late (blue),

583 true late (purple), unclassified (grey). We have also annotated additional genomic

584 features such as TATA boxes and ncRNA (white). 
Supplemental Fig. 5-2 Pol III machinery recruitment to the HSV-1 genome postviral genome replication

588 Human fibroblasts were infected with KOS (WT) HSV-1 for 4 or 6h. ChIP-Seq was

589 performed for POLR3A, POLR2A, TBP, GTF3A, GTF3C5, BRF1, and BRF2. Data is the

590 average of biological duplicates and normalized for sequencing depth and viral genome

591 copy number. Traces of binding to the unique long (UL), joint, and unique short (US)

592 regions of the HSV-1 genome (KT899744.1 assembly). Y-axes maximum and minimum

593 values are listed within brackets. Viral CDS are listed below, with colors indicating

594 transcriptional class: immediate early (yellow), early (green), leaky late (blue), true late

595 (purple), unclassified (grey). We have also annotated additional genomic features such 596 as TATA boxes and ncRNA (white).

\section{Fig. 6 Unique GTF context of viral Pol III binding}

599 Human fibroblasts were infected with KOS (WT) HSV-1 for 2 h. ChIP-Seq was 600 performed for POLR3A, TBP, GTF3C1, GTF3C5, GTF3C6, GTF3A, BRF2, POLR2A, 601 and ICP4. Data for GTF3C2, GTF3C3, GTF3C4 is not shown, as we did not observe 602 recruitment to the viral genome (Fig. 6-1). Data is normalized and presented as in Fig. 603 5. Yellow or grey boxes were used to highlight regions of Pol II-independent or 604 coincident binding, respectively.

606 Supplemental Fig. 6-1 Binding of Pol III machinery to canonical cellular targets.

607 ATAC-Seq or ChIP-Seq for POLR3A, TBP, BRF1, GTF3C5, GTF3A, BRF2, and 608 POLR2A was performed on uninfected human fibroblasts. Heatmaps are from $2 \mathrm{~kb}$ 
609 upstream of TSS to $2 \mathrm{~kb}$ downstream of TES for loci, and " $\mathrm{n}$ " indicates the number of

610 loci contained within each heatmap. y-axes are mapped reads per billion total reads

611 (MR/BTR). Models of canonical Pol III promoter architecture was adapted from (19).

612

613 Supplemental Fig. 6-2 TFIIIC complex recruitment to canonical cellular targets.

614 ATAC-Seq or ChIP-Seq for GTF3C1, GTF3C2, GTF3C3, GTF3C4, GTF3C5, and

615 GTF3C6 was performed on uninfected human fibroblasts. Heatmaps are from $2 \mathrm{~kb}$

616 upstream of TSS to $2 \mathrm{~kb}$ downstream of TES for loci, and " $\mathrm{n}$ " indicates the number of

617 loci contained within each heatmap. y-axes are mapped reads per billion total reads

618 (MR/BTR). Models of canonical Pol III promoter architecture was adapted from (19).

Supplemental Fig. 6-3 TFIIIC complex recruitment to the HSV-1 genome during

\section{1 early infection}

622 Human fibroblasts were infected with KOS (WT) HSV-1 for 2 h. ChIP-Seq was

623 performed for POLR3A, POLR2A, GTF3C1, GTF3C2, GTF3C3, GTF3C4, GTF3C5, and

624 GTF3C6. Data is the average of biological duplicates and normalized for sequencing

625 depth and viral genome copy number. Traces of binding to the unique long (UL), joint,

626 and unique short (US) regions of the HSV-1 genome (KT899744.1 assembly). Y-axes

627 maximum and minimum values are listed within brackets. Viral CDS are listed below,

628 with colors indicating transcriptional class: immediate early (yellow), early (green), leaky

629 late (blue), true late (purple), unclassified (grey). We have also annotated additional

630 genomic features such as TATA boxes and ncRNA (white). 
632 Fig. 7 Model of HSV-1 Pol II and III Modulation during lytic infection

633 During lytic infection, HSV-1 alters host Pol II recruitment and induces depletion

634 from host mRNA in favor of host tRNA and viral mRNA. With 12 hours, these changes

635 result in a 2-fold increase in total abundance of tRNA and a 10-fold increase in nascent

636 levels of tRNA. In contrast, host mRNA decreases to only $10 \%$ of the total present in the

637 cell. These phenomena are mediated distinctly. Depletion of Pol II from mRNA

638 promoters scales with viral genome replication and ICP4. Recruitment of Pol II to tRNA

639 promoters, and their subsequent upregulation, is caused by entry of the viral genome

640 and synthesis of viral transcripts - with no dependence on exactly which transcripts are

641 made.

642

643 


\section{METHODS:}

645

646 Cells and viruses

647 Vero (African green monkey kidney), U2OS (human osteosarcoma), and MRC5 (human

648 fetal lung) cells were obtained from and propagated as recommended by ATCC.

649 Viruses used in this study include HSV-1 mutants: n199 (ICP22 null, (62)), n212 (ICP0

650 null, (63)), d99 (ICP0 null, ((64))), n12 (ICP4 null, (65)), d120 (ICP4 null, (34)), 5 dl1.2

651 (ICP27 null, (66)), d92 (ICP4/27 null, (67)), d109 (ICP0/ICP4/ICP22/ICP27/ICP47 null,

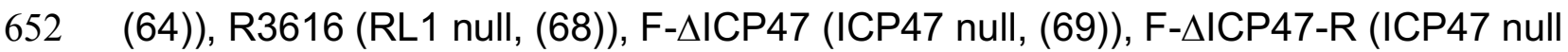
653 revertant, (69)), hp66 (UL30 null, (70)), and wild type HSV-1 (KOS, (71)). n199, R3616, $654 \mathrm{~F}-\Delta \mathrm{ICP} 47$, and KOS were prepared and titered in Vero cells. Other virus stocks were 655 prepared and titered in the following Vero-based complementing cell lines: E5 (ICP4+, 656 n12, d120-complementing, (72)); E11 (ICP4/ICP27+, 5dl1.2, d92-complementing, (67));

657 F06 (ICP4/ICP27/ICP0+, d109-complementing, (73)); U2OS (n212, d99-

658 complementing), POLB3 (hp66-complementing, (70)). We thank Don Coen (hp66,

659 POLB3), David A. Leib (R3616), and Anthony St. Leger (F- $\Delta \mathrm{ICP} 47, \mathrm{~F}-\Delta \mathrm{ICP} 47-\mathrm{R})$ for 660 their kind gift of viruses or cells. 


\section{Antibodies}

664 A list of all antibodies and the amount used per technique is located in Table 1.

666 Table 1. Antibodies Used in Study

\begin{tabular}{|c|c|c|c|c|}
\hline \multirow[b]{2}{*}{ Target } & \multirow[b]{2}{*}{ Antibody } & \multicolumn{3}{|c|}{ Amount/Dilution Used } \\
\hline & & ChIP-Seq & Immunofluorescence & $\begin{array}{l}\text { Western } \\
\text { Blot }\end{array}$ \\
\hline$\alpha$-Tubulin & AbCam \#ab7291 & $\mathrm{N} / \mathrm{A}$ & N/A & $1: 5000$ \\
\hline Vinculin & Abcam \#ab129002 & $\mathrm{N} / \mathrm{A}$ & $\mathrm{N} / \mathrm{A}$ & $1: 15000$ \\
\hline GAPDH & ThermoFisher \#AM4300 & $\mathrm{N} / \mathrm{A}$ & $\mathrm{N} / \mathrm{A}$ & $1: 5000$ \\
\hline POLR2A & AbCam \#ab5408 & $5 \mu \mathrm{g} / \mathrm{IP}$ & $1: 500$ & $\mathrm{~N} / \mathrm{A}$ \\
\hline POLR2A & SantaCruz \#sc899 & $\mathrm{N} / \mathrm{A}$ & $\mathrm{N} / \mathrm{A}$ & $1: 500$ \\
\hline TBP & AbCam \#ab51841 & $25 \mu \mathrm{g} / \mathrm{IP}$ & $\mathrm{N} / \mathrm{A}$ & $1: 1500$ \\
\hline POLR3A & AbCam \#ab96328 & $10 \mu \mathrm{g} / \mathrm{IP}$ & $1: 300$ & $\mathrm{~N} / \mathrm{A}$ \\
\hline POLR3A & CST \#12825S & $\mathrm{N} / \mathrm{A}$ & $\mathrm{N} / \mathrm{A}$ & $1: 500$ \\
\hline POLR3B & AbCam \#ab137030 & N/A & N/A & $1: 250$ \\
\hline POLR3C & Bethyl \#A303-064A-M & N/A & N/A & $1: 250$ \\
\hline POLR3D & AbCam \#ab86786 & $\mathrm{N} / \mathrm{A}$ & $\mathrm{N} / \mathrm{A}$ & $1: 500$ \\
\hline POLR3E & Sigma \#HPA041477 & $\mathrm{N} / \mathrm{A}$ & $\mathrm{N} / \mathrm{A}$ & $1: 125$ \\
\hline POLR3F & Abcam \#ab180501 & $\mathrm{N} / \mathrm{A}$ & $\mathrm{N} / \mathrm{A}$ & $1: 500$ \\
\hline BRF1 & SantaCruz \#sc-390821 & $10 \mu \mathrm{g} / \mathrm{IP}$ & $1: 200$ & $1: 100$ \\
\hline BRF2 & SantaCruz \#sc-390312 & $10 \mu \mathrm{g} / \mathrm{IP}$ & $\mathrm{N} / \mathrm{A}$ & $1: 100$ \\
\hline GTF3A & Abcam \#ab254632 & $1 \mu \mathrm{g} / \mathrm{IP}$ & $\mathrm{N} / \mathrm{A}$ & $1: 250$ \\
\hline GTF3C1 & Novus Biologicals \#NB100-60657 & $10 \mu \mathrm{g} / \mathrm{IP}$ & $\mathrm{N} / \mathrm{A}$ & $1: 500$ \\
\hline GTF3C2 & AbCam \#ab89113 & $7.5 \mu \mathrm{g} / \mathrm{IP}$ & $\mathrm{N} / \mathrm{A}$ & $1: 100$ \\
\hline GTF3C3 & SantaCruz \#sc-101176 & $7.5 \mu \mathrm{g} / \mathrm{IP}$ & N/A & $1: 100$ \\
\hline GTF3C4 & Sigma \#HPA069369 & $3 \mu \mathrm{g} / \mathrm{IP}$ & $\mathrm{N} / \mathrm{A}$ & $1: 500$ \\
\hline GTF3C5 & Bethyl \#A301-242A & $3 \mu \mathrm{g} / \mathrm{IP}$ & $1: 500$ & $1: 500$ \\
\hline GTF3C6 & ThermoFisher \#PA5-63948 & $7.5 \mu \mathrm{g} / \mathrm{IP}$ & $\mathrm{N} / \mathrm{A}$ & $1: 100$ \\
\hline
\end{tabular}


669 Oligos

670 A list of all single stranded oligos used for either qPCR or Northern Blot is located in

671 Table 2.

672

673 Table 2. Oligos Used in Study

\begin{tabular}{|c|c|c|}
\hline Name & Sequence (5' to $3^{\prime}$ ) & Used for \\
\hline U6 snRNA & $\begin{array}{l}\text { GAATTTGCGTGTCATCCTTGCGCAGGG } \\
\text { GCCATGCTAA }\end{array}$ & Northern Blot \\
\hline 7SL & AACTTAGTGCGGACACCCGATC & Northern Blot \\
\hline 7SK & CGGGGAAGGTCGTCCTCTTC & Northern Blot \\
\hline 18S rRNA F & GTAACCCGTTGAACCCCATT & RT-qPCR \\
\hline $18 S$ rRNA $R$ & CСATCCAATCGGTAGTAGCG & RT-qPCR \\
\hline 45S pre-rRNA F & СTCCGTTATGGTAGCGCTGC & RT-qPCR \\
\hline 45S pre-rRNA R & GCGGAACCСTCGCTTCTC & RT-qPCR \\
\hline Pre-tRNA Leu-CAA-2-1 F & ATGGCCGAGTGGTCTAAGG & RT-qPCR \\
\hline Pre-tRNA Leu-CAA-2-1 R & ACCAGAAGACCCGAACACAG & RT-qPCR \\
\hline Pre-tRNA Tyr-GTA-5-3 F & СCTTCGATAGCTCAGCTGGT & RT-qPCR \\
\hline Pre-tRNA Tyr-GTA-5-3 R & CGACCTAAGGATGTCCACAAAT & RT-qPCR \\
\hline Pre-tRNA Arg-TCT-2-1 F & GGCTCTGTGGCGCAATGGATA & RT-qPCR \\
\hline Pre-tRNA Arg-TCT-2-1 R & TTCGAАCCCACAАCСTTTGAATTGCTC & RT-qPCR \\
\hline tRNA iMet-CAT-1 F & CTGGGCCCATAACCCAGAG & RT-qPCR \\
\hline tRNA iMet-CAT-1 R & TGGTAGCAGAGGATGGTTTC & RT-qPCR \\
\hline $5 S$ rRNA F & GGCCATACCACCCTGAACGC & RT-qPCR \\
\hline $5 S$ rRNA R & CAGCACCCGGTATTCCCAGG & RT-qPCR \\
\hline 7SL F & САAАACTCCCGTGCTGATCA & RT-qPCR \\
\hline 7SL R & GGCTGGAGTGCAGTGGCTAT & RT-qPCR \\
\hline U6 snRNA F & GGAATCTAGAACATATACTAAAATTGGAAC & RT-qPCR \\
\hline U6 snRNA R & GGAACTCGAGTTTGCGTGTCATCCTTGCGC & RT-qPCR \\
\hline tRNA Sec F & GGCTGATCCTCAGTGGTC & RT-qPCR \\
\hline tRNA Sec R & GGTGGAATTGAACCACTC & RT-qPCR \\
\hline 7SK F & CGATCTGGTTGCGACATCTG & RT-qPCR \\
\hline 7SK R & CGTTCTCCTACAAATGGAC & RT-qPCR \\
\hline BC200 F & ATAGCTTGAGCCCAGGAGTT & RT-qPCR \\
\hline BC200 R & GСTTTGAGGGAAGTTACGCTTAT & RT-qPCR \\
\hline
\end{tabular}




\begin{tabular}{|l|l|l|}
\hline Name & Sequence (5' to 3') & Used for \\
\hline PretRNA-Arg-TCT-2-1 & TGCTCTATTCGTCACTAGAAGTCC & Northern Blot \\
\hline Arg Unfolder & GCTATCCATTGCGCCACAGAGCC & Northern Blot \\
\hline PretRNA-Ile-TAT-1-1 & CCGCTCGCACTGTCATATAAGTACC & Northern Blot \\
\hline Ile Unfolder & CTAACCGATTGCGCCACTGGAGC & Northern Blot \\
\hline PretRNA-Tyr-GTA-5-1 & GGATGTCTCCTGCTGAGGAAGTAGC & Northern Blot \\
\hline Tyr Unfolder & TCCTTCGAGCCGGAATCGAACCAGCGACC & Northern Blot \\
\hline Mature tRNA-Gly-GCC & TGGTGCATTGGCCGGGAATCGAACC & Northern Blot \\
\hline Gly Unfolder & ATTCTACCACTGAACCACCAATGC & Northern Blot \\
\hline Mature tRNA-Asp-GTC & TGGCTCCCCGTCGGGGAATCGAAC & Northern Blot \\
\hline Asp Unfolder & CACTCACCACTATACTAACGAGGA & Northern Blot \\
\hline $\mathbf{5 S}$ & CGGTATTCCCAGGCGGTCT & Northern Blot \\
\hline $\mathbf{5 . 8 5}$ & CAATGTGTCCTGCAATTCAC & Northern Blot \\
\hline
\end{tabular}

676 Viral infection

677 Confluent cell monolayers were infected with 10 PFU per cell. Virus was adsorbed in

678 tricine-buffered saline (TBS) for $1 \mathrm{hr}$ at room temperature. Viral inoculum was removed,

679 and cells were washed quickly with TBS before adding $2 \%$ FBS media. 0 hour time

680 point was considered after adsorption of infected monolayers when cells were place at

$68137^{\circ} \mathrm{C}$ to incubate.

682

683 qPCR Quantification of mRNA.

684 Cell monolayers were collected by aspirating supernatant and washing twice with TBS.

685 Cells were scraped into $1 \mathrm{~mL}$ TBS and pelleted, supernatant was discarded. RNA was

686 isolated using the RNAqueous Micro Kit (ThermoFisher cat. no. AM1931). cDNA was

687 generated from $500 \mathrm{ng}$ total RNA, as quantified using the Agilent RNA 6000 Nano Kit.

688 RNA was reverse transcribed with 20 units Riboguard RNase inhibitor, 2.5 uM Random

689 decamer primer (Invitrogen cat no. 5722G), 1004 units MMLV-HP reverse 
690 transcriptase, $10 \mathrm{mM}$ dithiothreitol, $2.5 \mathrm{mM}$ dNTPs, and 1x reaction buffer (Epicentre cat

691 no. RT80125K and RG90910K). RNA and random decamer primer were first incubated

692 at $85^{\circ} \mathrm{C}$ for 3 minutes and then incubated on ice for 2 minutes during which remaining

693 reaction components were added. The entire reaction was incubated at $65^{\circ} \mathrm{C}$ for 2

694 minutes and then $37^{\circ} \mathrm{C}$ for 1 hour. To heat inactivate components the cDNA was

695 incubated at $85^{\circ} \mathrm{C}$ for 5 minutes. Standard curves were generated using purified KOS or 696 human genome stocks.

698 Western Blot

699 At the indicated times postinfection, proteins were isolated from cells using Laemmli

700 SDS sample buffer and Western blotting was carried out using the primary antibodies

701 listed in table 1. Blot were probed with secondary antibodies-IRDye goat anti rabbit or

702 goat anti-mouse 680/800 — at a 1:15000 dilution. The intensity of gel bands was

703 quantified using the Odyssey CLx system machine and Image Studio program. Band

704 intensities were normalized to loading controls including GAPDH, alpha-tubulin

705 (TUB4A), or Vinculin (VCL) detected from the same sample.

707 Northern Blot

708 Total RNA was isolated from cells using TRIzol (Invitrogen), following manufacturer's

709 instructions. RNA was quantified using Agilent RNA 6000 Nano kit. 5-20 $\mu$ g of RNA was

710 loaded into 10\% TBE-Urea Polyacrylamide Gel, transferred to Hybond-N+ (Sigma)

711 membranes using the Owl ${ }^{\mathrm{TM}}$ HEP Series Semidry Electroblotting Systems

712 (ThermoFisher). Blots were pre-hybridized in ExpressHyb buffer (Takara Bio) at $37^{\circ} \mathrm{C}$ 
713 for 1 hour. If using radiolabeled probes specific to tRNAs, 50 nM unfolder oligo (see

714 Table 2) was included in the pre-hybridization step, as described in (74). Probes were

715 generated by end-labeling oligos listed in Table 2 using T4 PNK and [g-32P]- ATP.

716 Probes were hybridized to the blots in ExpressHyb buffer at $37^{\circ} \mathrm{C}$ overnight. If using

717 radiolabeled probes specific to tRNAs, $25 \mathrm{nM}$ unfolder oligo was included in the

718 hybridization step. After hybridization, blots were washed four time in 2x SSC [0.3M

$719 \mathrm{NaCl}, 0.03 \mathrm{M}$ Trisodium Citrate $\mathrm{pH} 7.0], 0.05 \%$ SDS and 2 times in $0.1 \times \mathrm{SSC}, 0.1 \%$

720 SDS. Blots were exposed and quantified using the Typhoon Biomolecular Imagine

721 (Amersham). Blots were stripped in 1\% SDS, 0.1x SSC, $40 \mathrm{mM}$ Tris pH 8 for four 10

722 minute washes at $70^{\circ} \mathrm{C}$. Northern blot quantifications were normalized to $5.8 \mathrm{~S}$ as a

723 loading control.

725 Immunofluorescence

$7261.7 \times 10^{5}$ Vero cells were infected as described above. Coverslips were fixed at

727 indicated time point with $3.7 \%$ paraformaldehyde. EdC labeling of viral replication

728 compartments, click chemistry, and immunofluorescence were conducted as previously

729 described $(42,75)$. Cellular DNA was stained with 1:2000 Hoescht, and

730 immunofluorescence was carried out using antibodies listed in table 1 and 594-

731 conjugated secondary antibodies (Santa Cruz, 1:500). Images were taken using an

732 Olympus Fluoview FV1000 confocal microscope. Images are representative of

733 biological duplicate experiments. 
736 Protocol was modified from $(29,35)$, with the following modifications. $7 \times 10^{7}$ MRC5

737 cells were infected as described above and total RNA was isolated from cells using

738 TRIzol (Invitrogen), following manufacturer's instructions. Total RNA extracted from two

739 biological replicates was spiked with in vitro transcribed E. coli tRNA-Lys, E. coli tRNA-

$740 \mathrm{Tyr}$, and S. cerevisiae tRNA-Phe transcripts at 0.01 pmol IVT tRNAs per $\mu \mathrm{g}$ total RNA.

741 RNA was deacylated in $0.1 \mathrm{M}$ Tris- $\mathrm{HCl}, \mathrm{pH} 9$ at $37^{\circ} \mathrm{C}$ for $45 \mathrm{~min}$, ethanol purified, and

742 then dephosphorylated with PNK. Deacylated and dephosphorylated RNAs were

743 purified with a mirVANA small RNA purification kit (Ambion). RNAs were demethylated

744 in $300 \mathrm{mM} \mathrm{NaCl}, 50 \mathrm{mM}$ MES pH 5, $2 \mathrm{mM} \mathrm{MgCl} 2,50 \mu \mathrm{M}$ ferrous ammonium sulfate,

$745300 \mu \mathrm{M}$ 2-ketoglutarate, $2 \mathrm{mM}$ L-ascorbic acid, $50 \mu \mathrm{g} / \mathrm{ml}$ BSA, $1 \mathrm{U} / \mu \mathrm{l}$ SUPERasin, $2 \mathrm{X}$

746 molar ratio of wt AlkB, and 4X molar ratio of D135S AlkB for 2 hours at room

747 temperature. Ni-NTA cation exchange purified His-tagged wild-type and D135S AlkB.

748 Reaction was quenched with 5 mM EDTA and purified with Trizol LS reagent. Two

749 TGIRT reactions from each biological replicate was performed, these are considered

750 technical replicates. In total each sample condition is an average of 4 data points,

751 consisting of two biological replicates containing technical duplicates. $100 \mathrm{ng}$

752 demethylated small RNAs was used for library prep with a TGIRT Improved Modular

753 Template- Switching RNA-seq Kit (InGex) following the manufacturer's instructions.

754 PCR amplification was performed with Phusion polymerase (Thermo Fisher) with

755 Illumina multiplex and barcoded primers. Libraries were quantified using the Agilent

756 DNA $7500 \mathrm{Kit}$, and samples were mixed together at equimolar concentration. Samples

757 were size selected for $150-250$ bp fragments using the Pippin system. Illumina NextSeq 
758550 platform was used to generate $75 \mathrm{bp}$ PE reads and carried out at the Tufts

759 University Core Facility.

760

761 4SU-Sequencing

$7622 \times 10^{6}$ MRC5 cells were infected with wild-type HSV-1 (KOS) as described above. At

763 indicated time (hpi) 500 uM 4SU (Sigma) was added to cell culture medium. 15 minutes

764 post-4SU addition, cells were collected and RNA extracted using miRNeasy kit

765 (Qiagen). 50-100 ug total RNA was biotinylated in $10 \mathrm{mM}$ Tris pH 7.4, 1 mM EDTA, 0.2

$766 \mathrm{mg} / \mathrm{mL}$ EZ-link Biotin-HPDP (ThermoFisher). Unbound biotin was removed by

767 performing a chloroform:isoamyl alcohol extraction using MaXtract High Density tubes

768 (Qiagen). RNA was isopropanol precipitated and resuspended in water. Biotinylated

769 RNA was bound 1:1 to Dynabeads My One Streptavidin T1 equilibrated in $10 \mathrm{mM}$ Tris

$770 \mathrm{pH} 7.5,1 \mathrm{mM}$ EDTA, $2 \mathrm{M} \mathrm{NaCl}$. Bound beads were washed three times with $5 \mathrm{mM}$ Tris

$771 \mathrm{pH}$ 7.5, $1 \mathrm{mM}$ EDTA, $1 \mathrm{M} \mathrm{NaCl}$. 4SU-RNA was eluted with $100 \mathrm{mM}$ DTT and isolated

772 using the RNeasy MinElute Cleanup Kit (Qiagen). RNA-Seq libraries were generated

773 using NEBNext Ultra Directional RNA Library Prep Kit for Illumina. Libraries were

774 quantified using the Agilent DNA 7500 Kit, and samples were mixed together at

775 equimolar concentration. One biological replicate was sequenced using the Illumina

776 HiSeq 2500 platform to generate $50 \mathrm{bp}$ SE reads and carried out at the Tufts University

777 Core Facility.

778

779 PolyA-Selected RNA-Seq 
780 Total RNA was harvested using the Ambion RNAqueous-4PCR kit and quantified using

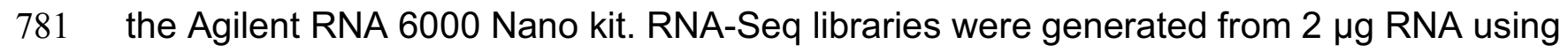

782 NEBNext Poly(A) mRNA Magnetic Isolation Module and NEBNext Ultra Directional RNA

783 Library Prep Kit for Illumina (NEB \#E7490 and \#E7420). Libraries were quantified using

784 the Agilent DNA $7500 \mathrm{Kit}$, and samples were mixed together at equimolar concentration.

785 Two biological replicates were sequenced using the Illumina HiSeq 2500 platform to

786 generate 50 bp SE reads and carried out at the Tufts University Core Facility.

788 Ribo-minus Total RNA-Seq

789 Total RNA was isolated from cells using TRIzol (Invitrogen), following manufacturer's

790 instructions. RNA was quantified using Agilent RNA 6000 Nano kit. ERCC spike-in

791 controls (ThermoFisher) were added to $500 \mathrm{ng}$ of total RNA and ribominus selection

792 was performed using the NEBNext ${ }^{2}$ rRNA Depletion Kit. RNA-Seq libraries were

793 generated using the NEBNext Ultra Directional RNA Library Prep Kit for Illumina.

794 Libraries were quantified using the Agilent DNA $7500 \mathrm{Kit}$, and samples were mixed

795 together at equimolar concentration. One biological replicates was sequenced using the

796 Illumina NextSeq550 platform to generate 75 bp PE reads and carried out at the Tufts

797 University Core Facility.

799 ChIP-Sequencing

800 ChIP-Seq was performed on mock-infected or infected MRC5 cells as described

801 previously (11), with antibodies listed in Table 1. Libraries were quantified using the

802 Agilent DNA $7500 \mathrm{Kit}$, and samples were mixed together at equimolar concentration. 
803 For each IP two biological replicates were sequenced, for input at least two biological

804 replicates were sequenced using the Illumina HiSeq 2500 platform was used to

805 generate 50 bp SE reads and carried out at the Tufts University Core Facility.

806

807 ATAC-Sequencing

808 We adapted the protocol from Buenrostro et al. (2013), and previously published the

809 data see SRA\# PRJNA553559 (11).

810

811 Data Availability

812 All datasets are publicly available at:

813 ATAC-Seq of WT (KOS) and ICP4 null (n12) HSV-1 Productive Infection in MRC5 cells

814 SRA: PRJNA553559

815 ICP4 ChIP-Seq of WT (KOS) HSV-1 Productive Infection in MRC5 cells SRA:

816 PRJNA553563

817 HSV-1 4SU-Seq SRA: PRJNA692715

818 HSV-1 POL3 Machinery-ChIP-Seq SRA: PRJNA693164

819 HSV-1 DM-tRNA-Seq SRA: PRJNA692681

820 HSV-1 GTF3C Complex-ChIP-Seq SRA: PRJNA732084

821 ChIP-Seq for POLR2A in HSV-1 infected human fibroblasts SRA: PRJNA732212

822 PolyA-Selected RNA-Seq in HSV-1 infected human fibroblast cells SRA: PRJNA732134

823 Total RNA-Seq in HSV-1 Infected Human Fibroblasts SRA: PRJNA732091

824

825 Bioinformatic Analysis 
826 Data was uploaded to the Galaxy web platform, and we used the public server at

827 usegalaxy.org to analyze the data (76).

831 We quantified mature and premature tRNA in 4SU-Seq and DM-tRNA-Seq datasets

832 similar to the analysis described in (29). To discriminate between pre- and mature-tRNA

833 species we first mapped sequencing data to an assembly of mature-tRNA sequences

834 wherein 5' and 3' leader sequences are absent, introns are spliced, and 3' CCA tails are

835 present. Unaligned reads were then mapped to a modified host (hg38) genome in which

836 tRNA genes were masked and instead appended as an additional chromosome

837 containing pre-tRNA sequences with introns, and 5' and 3' leader sequences. Mature

838 and premature-tRNA assemblies for hg38 were generated using the tRAX pipeline (77).

839 Mitochondrial and nuclear tRNA loci were based on GtRNAdb $(78,79)$ and tRNAscan-

840 SE (80) for hg38.

841 To assess mRNA levels, Ribominus Total or PolyA-selected RNA-Seq data was aligned

842 sequentially using HISAT2 to the human genome (hg38) and HSV-1 strain KOS

843 genome (KT899744.1) (81). FeatureCounts was performed using the KT89974.1 CDS

844 as the reference GFF (82). For PolyA-selected RNA-Seq raw counts were normalized

845 as mapped reads per billion total reads per kilobase (MR/BTR/Kb). For Ribominus Total

846 RNA-Seq raw counts were normalized as mapped reads per million ERCC spike-in

847 reads per kilobase (MR/MSI/Kb). 
850 For ChIP-Seq, data was first aligned using Bowtie2 (83) to the human genome (hg38),

851 and then unaligned reads were mapped to the HSV-1 strain KOS genome

852 (KT899744.1). A modified version of the KT899744.1 genome was created, removing

853 one copy of the repeat joint region $(\Delta 1-9603, \Delta 125,845-126,977, \Delta 145,361-151,974)$.

854 Bam files were visualized using DeepTools bamcoverage (84) with a bin size of 1 to

855 generate bigwig files. Data was viewed in IGV viewer and exported as EPS files. Bigwig

856 files were normalized for sequencing depth and genome quantity. Mapped reads were

857 multiplied by the 'norm factor' which was calculated as the inverse of (Input cellular

858 reads)/(Input cellular+viral mapped reads (TMR) )×Billion sample TMR or (Input viral

859 reads)/TMR $\times$ Million sample TMR. ChIP-Seq experiments were repeated for a total of 2-

8603 biological replicates. The normalized bigwig files were averaged between replicates.

861 Heatmaps and gene profiles were generated using MultiBigwigSummary on normalized

862 cellular bigwig files to all UCSC annotated mRNAs or high-confident tRNA loci from

863 GtRNAdb $(78,79)$. Gene profiles and heatmaps were plotted using plotProfile and

864 plotHeatmap (84).

865 For ATAC-Seq, data was first aligned using Bowtie2 (83) to the mitochondrial genome.

866 Unaligned reads were sequentially mapped to the human genome (hg38), and the HSV-

8671 strain KOS genome (KT899744.1) with the following parameters: -no-unal -local -

868 very-sensitive-local -nodiscordant -no-mixed -contain -overlap -dovetail -phred33.

869 Bam files were visualized using DeepTools bamcoverage (84) with a bin size of 1 to

870 generate bigwig files. Data was viewed in IGV viewer and exported as EPS files.

871 Cellular bigwig files were normalized for sequencing depth (excluding mitochondrial 
872 mapped reads), the $y$-axes values are mapped reads per billion reads. The normalized

873 bigwig files were averaged between two biological replicates. Heatmaps and gene

874 profiles were generated using MultiBigwigSummary (84) on normalized cellular bigwig

875 files to all UCSC annotated mRNAs. Gene profiles and heatmaps were plotted using

876 plotProfile and plotHeatmap (231). 


\section{REFERENCES}

878 1. J. C. Alwine, W. L. Steinhart, C. W. Hill, Transcription of herpes simplex type 1

879 DNA in nuclei isolated from infected HEp-2 and KB cells. Virology 60, 302-307

880

881

882

883

884 (1974).

2. R. Honess, B. Roizman, Regulation of herpesvirus macromolecular synthesis, I. Cascade regulation of the synthesis of three groups of viral proteins. J Virol 14, 8-19 (1974).

3. R. Honess, B. Roizman, Regulation of herpesvirus macromolecular synthesis: Sequential transition of polypeptide synthesis requires functional viral polypeptides. Proc Natl Acad Sci USA 72, 1276-1280 (1975).

4. S. Dremel, N. DeLuca, Genome replication affects transcription factor binding mediating the cascade of herpes simplex virus transcription. Proc Natl Acad Sci USA 116, 3734-3739 (2019). Academy of Sciences 84, 1926-1930 (1987).

6. A. A. Oroskar, G. S. Read, Control of mRNA stability by the virion host shutoff function of herpes simplex virus. Journal of Virology 63, 1897-1906 (1989).

7. P. Feng, D. N. Everly, G. S. Read, mRNA Decay during Herpesvirus Infections: Interaction between a Putative Viral Nuclease and a Cellular Translation Factor. Journal of Virology 75, 10272-10280 (2001).

8. R. Abrisch, T. Eidem, P. Yakovchuk, J. Kugel, J. Goodrich, Infection by herpes simplex virus 1 causes near-complete loss of RNA polymerase II occupancy on the host cell genome. J Virol 90, 2503-2513 (2015).

902

903

904

9. C. Birkenheuer, C. Danko, J. Baines, Herpes simplex virus 1 dramatically alters loading and positioning of RNA polymerase II on host genes early in infection. $J$ Virol 92, e02184-02117 (2018).

10. D. McSwiggen et al., Evidence for DNA-mediated nuclear compartmentalization distinct from phase separation. eLife 8, e47098 (2019).

11. S. Dremel, N. DeLuca, Herpes simplex viral nucleoprotein creates a competitive transcriptional environment facilitating robust viral transcription and host shut off. eLife 8, e51109 (2019).

12. R. G. Roeder, W. J. Rutter, Multiple forms of DNA-dependent RNA polymerase in eukaryotic organisms. Nature 224, 234-237 (1969).

13. R. G. Roeder, Lasker Basic Medical Research Award. The eukaryotic transcriptional machinery: complexities and mechanisms unforeseen. Nat Med $\mathbf{9}$, 1239-1244 (2003).

14. R. Weinmann, T. G. Brendler, H. J. Raskas, R. G. Roeder, Low molecular weight viral RNAs transcribed by RNA polymerase III during adenovirus 2 infection. Cell 7, 557-566 (1976).

15. M. D. Rosa, E. Gottlieb, M. R. Lerner, J. A. Steitz, Striking similarities are exhibited by two small Epstein-Barr virus-encoded ribonucleic acids and the adenovirus-associated ribonucleic acids VAI and VAll. Mol Cell Biol 1, 785-796 $920 \quad$ (1981). 
921 16. E. R. Feldman et al., Virus-encoded microRNAs facilitate gammaherpesvirus

922

923

924

925

926

927

928

929

930

931

932

933

934

935

936

937

938

939

940

941

942

943

944

945

946

947

948

949

950

951

952

953

954

955

956

957

958

959

960

961

962

963

964

965 latency and pathogenesis in vivo. mBio 5, e00981-00914 (2014).

17. H. P. Bogerd et al., A mammalian herpesvirus uses noncanonical expression and processing mechanisms to generate viral MicroRNAs. Mol Cell 37, 135-142 (2010).

18. K. W. Diebel, A. L. Smith, L. F. van Dyk, Mature and functional viral miRNAs transcribed from novel RNA polymerase III promoters. RNA 16, 170-185 (2010).

19. A. J. Oler et al., Human RNA polymerase III transcriptomes and relationships to Pol II promoter chromatin and enhancer-binding factors. Nat. Struct. Biol 17, 620629 (2010).

20. Z. Moqtaderi et al., Genomic binding profiles of functionally distinct RNA polymerase III transcription complexes in human cells. Nat. Struct. Biol 17, 635641 (2010).

21. D. Raha et al., Close association of RNA polymerase II and many transcription factors with Pol III genes. Proc Natl Acad Sci USA 107, 3639-3644 (2010).

22. I. Listerman, A. S. Bledau, I. Grishina, K. M. Neugebauer, Extragenic Accumulation of RNA Polymerase II Enhances Transcription by RNA Polymerase III. PLoS Genetics 3, e212 (2007).

23. R. B. Gaynor, L. T. Feldman, A. J. Berk, Transcription of class III genes activated by viral immediate early proteins. Science 230, 447-450 (1985).

24. W. K. Hoeffler, R. G. Roeder, Enhancement of RNA polymerase III transcription by the E1A gene product of adenovirus. Cell 41, 955-963 (1985).

25. B. Panning, J. R. Smiley, Activation of RNA polymerase III transcription of human Alu repetitive elements by adenovirus type 5: requirement for the E1b 58kilodalton protein and the products of E4 open reading frames 3 and 6 . Mol Cell Biol 13, 3231-3244 (1993).

26. Z. A. Felton-Edkins, R. J. White, Multiple mechanisms contribute to the activation of RNA polymerase III transcription in cells transformed by papovaviruses. J Biol Chem 277, 48182-48191 (2002).

27. Z. A. Felton-Edkins et al., Epstein-Barr virus induces cellular transcription factors to allow active expression of EBER genes by RNA polymerase III. J Biol Chem 281, 33871-33880 (2006).

28. J. Karijolich, E. Abernathy, B. A. Glaunsinger, Infection-Induced Retrotransposon-Derived Noncoding RNAs Enhance Herpesviral Gene Expression via the NF-kB Pathway. PLOS Pathogens 11, e1005260 (2015).

29. J. M. Tucker, A. M. Schaller, I. Willis, B. A. Glaunsinger, Alteration of the Premature tRNA Landscape by Gammaherpesvirus Infection. mBio 11, e0266402620 (2020).

30. K. L. Jang, D. S. Latchman, HSV infection induces increased transcription of Alu repeated sequences by RNA polymerase III. FEBS Lett 258, 255-258 (1989).

31. B. Panning, J. R. Smiley, Activation of RNA polymerase III transcription of human Alu elements by herpes simplex virus. Virology 202, 408-417 (1994).

32. K. M. Eidson, W. E. Hobbs, B. J. Manning, P. Carlson, N. A. DeLuca, Expression of Herpes Simplex Virus ICPO Inhibits the Induction of Interferon-Stimulated Genes by Viral Infection. Journal of Virology 76, 2180 (2002). 
966

967

968

969

970

971

972

973

974

975

976

977

978

979

980

981

982

983

984

985

986

987

988

989

990

991

992

993

994

995

996

997

998

999

1000

1001

1002

1003

1004

1005

1006

1007

1008

1009

1010
33. X.-D. Li et al., Pivotal roles of cGAS-cGAMP signaling in antiviral defense and immune adjuvant effects. Science (New York, N.Y.) 341, 1390-1394 (2013).

34. N. Deluca, A. McCarthy, P. Schaffer, Isolation and characterization of deletion mutants of herpes simplex virus type 1 in the gene encoding immediate-early regulatory protein ICP4. J Virol 56, 558-570 (1985).

35. G. Zheng et al., Efficient and quantitative high-throughput tRNA sequencing. Nat Methods 12, 835-837 (2015).

36. H. A. Saffran, J. M. Pare, J. A. Corcoran, S. K. Weller, J. R. Smiley, Herpes simplex virus eliminates host mitochondrial DNA. EMBO Rep 8, 188-193 (2007).

37. Andreas H. Ehrensberger, Gavin P. Kelly, Jesper Q. Svejstrup, Mechanistic Interpretation of Promoter-Proximal Peaks and RNAPII Density Maps. Cell 154, 713-715 (2013).

38. M. Campbell, J. Palfreyman, C. Preston, Identification of herpes simplex virus DNA sequences which encode a trans-acting polypeptide responsible for stimulation of immediate early transcription. J Mol Biol 180, 1-19 (1984).

39. W. Batterson, B. Roizman, Characterization of the herpes simplex vironassociated factor responsible for the induction of alpha genes. $J$ Virol 46, 371377 (1983).

40. A. Wilson, K. LaMarco, M. Peterson, W. Herr, The VP16 accessory protein HCF is a family of polypeptides processed from a large precursor protein. Cell 74, 115-125 (1993).

41. T. Kristie, P. Sharp, Purification of the cellular $\mathrm{C} 1$ factor required for the stable recognition of the Oct-1 homeodomain by the herpes simplex virus alpha-transinduction factor (VP16). J Biol Chem 268, 6525-6534 (1993).

42. J. Dembowski, N. DeLuca, Selective recruitment of nuclear factors to productively replicating herpes simplex virus genomes. PLoS Pathog 11, e1004939 (2015).

43. J. Alwine, W. Steinhart, C. Hill, Transcription of herpes simplex type 1 DNA in nuclei isolated from infected HEp-2 and KB cells. Virology 60, 302-307 (1974).

44. A. van Weringh et al., HIV-1 modulates the tRNA pool to improve translation efficiency.

45. A. C. Stabell et al., Non-human Primate Schlafen11 Inhibits Production of Both Host and Viral Proteins. PLOS Pathogens 12, e1006066 (2016).

46. M. Li et al., Codon-usage-based inhibition of HIV protein synthesis by human schlafen 11. Nature 491, 125-128 (2012).

47. J. J. Chiang et al., Viral unmasking of cellular $5 S$ rRNA pseudogene transcripts induces RIG-I-mediated immunity. Nat Immunol 19, 53-62 (2018).

48. H. Zhu, C. Zheng, The Race between Host Antiviral Innate Immunity and the Immune Evasion Strategies of Herpes Simplex Virus 1. Microbiology and Molecular Biology Reviews 84, e00099-00020 (2020).

49. A. Gerber, K. Ito, C.-S. Chu, R. G. Roeder, Gene-Specific Control of tRNA Expression by RNA Polymerase II. Molecular Cell 78, 765-778.e767 (2020).

50. M. Torrent, G. Chalancon, N. S. de Groot, A. Wuster, M. Madan Babu, Cells alter their tRNA abundance to selectively regulate protein synthesis during stress conditions. Science Signaling 11, eaat6409 (2018). 
1011 51. L. Vastag, E. Koyuncu, S. L. Grady, T. E. Shenk, J. D. Rabinowitz, Divergent

1012

1013

1014

1015

1016

1017

1018

1019

1020

1021

1022

1023

1024

1025

1026

1027

1028

1029

1030

1031

1032

1033

1034

1035

1036

1037

1038

1039

1040

1041

1042

1043

1044

1045

1046

1047

1048

1049

1050

1051

1052

1053

1054 Effects of Human Cytomegalovirus and Herpes Simplex Virus-1 on Cellular Metabolism. PLOS Pathogens 7, e1002124 (2011).

52. H. F. Burnett, T. E. Audas, G. Liang, R. R. Lu, Herpes simplex virus-1 disarms the unfolded protein response in the early stages of infection. Cell stress \& chaperones 17, 473-483 (2012).

53. S. Smith, S. K. Weller, HSV-I and the cellular DNA damage response. Future virology 10, 383-397 (2015).

54. J. G. Stevens, E. K. Wagner, G. B. Devi-Rao, M. L. Cook, L. T. Feldman, RNA complementary to a herpesvirus alpha gene mRNA is prominent in latently infected neurons. Science 235, 1056-1059 (1987).

55. L. Yeh, P. A. Schaffer, A novel class of transcripts expressed with late kinetics in the absence of ICP4 spans the junction between the long and short segments of the herpes simplex virus type 1 genome. J Virol 67, 7373-7382 (1993).

56. J. L. Umbach et al., MicroRNAs expressed by herpes simplex virus 1 during latent infection regulate viral mRNAs. Nature 454, 780-783 (2008).

57. R. A. Bohenzky, A. G. Papavassiliou, I. H. Gelman, S. Silverstein, Identification of a promoter mapping within the reiterated sequences that flank the herpes simplex virus type 1 UL region. J Virol 67, 632-642 (1993).

58. A. T. Dobson et al., Identification of the latency-associated transcript promoter by expression of rabbit beta-globin mRNA in mouse sensory nerve ganglia latently infected with a recombinant herpes simplex virus. J Virol 63, 3844-3851 (1989).

59. B. Gu, R. Kuddus, N. A. DeLuca, Repression of activator-mediated transcription by herpes simplex virus ICP4 via a mechanism involving interactions with the basal transcription factors TATA-binding protein and TFIIB. Mol Cell Biol 15, 3618-3626. (1995).

60. R. Rivera-Gonzalez, A. N. Imbalzano, B. Gu, N. A. Deluca, The role of ICP4 repressor activity in temporal expression of the IE-3 and latency-associated transcript promoters during HSV-1 infection. Virology 202, 550-564 (1994).

61. T. K. Soh et al., Temporal Proteomic Analysis of Herpes Simplex Virus 1 Infection Reveals Cell-Surface Remodeling via pUL56-Mediated GOPC Degradation. Cell Reports 33 (2020).

62. S. A. Rice, M. C. Long, V. Lam, P. A. Schaffer, C. A. Spencer, Herpes simplex virus immediate-early protein ICP22 is required for viral modification of host RNA polymerase II and establishment of the normal viral transcription program. $J$ Virol 69, 5550-5559. (1995).

63. W. Z. Cai, P. A. Schaffer, Herpes simplex virus type 1 ICP0 plays a critical role in the de novo synthesis of infectious virus following transfection of viral DNA. $J$ Virol 63, 4579-4589. (1989).

64. L. A. Samaniego, L. Neiderhiser, N. A. DeLuca, Persistence and expression of the herpes simplex virus genome in the absence of immediate-early proteins. $J$ Virol 72, 3307-3320. (1998).

65. N. DeLuca, P. Schaffer, Physical and functional domains of the herpes simplex virus transcriptional regulatory protein ICP4. J Virol 62, 732-743 (1988). 
1055

1056

1057

1058

1059

1060

1061

1062

1063

1064

1065

1066

1067

1068

1069

1070

1071

1072

1073

1074

1075

1076

1077

1078

1079

1080

1081

1082

1083

1084

1085

1086

1087

1088

1089

1090

1091

1092

1093

1094

1095

1096

1097

1098

1099
66. A. M. McCarthy, L. McMahan, P. A. Schaffer, Herpes simplex virus type 1 ICP27 deletion mutants exhibit altered patterns of transcription and are DNA deficient. $J$ Virol 63, 18-27. (1989).

67. L. A. Samaniego, A. L. Webb, N. A. DeLuca, Functional interactions between herpes simplex virus immediate-early proteins during infection: gene expression as a consequence of ICP27 and different domains of ICP4. J Virol 69, 5705-5715 (1995).

68. J. Chou, E. Kern, R. Whitley, B. Roizman, Mapping of herpes simplex virus-1 neurovirulence to gamma 134.5, a gene nonessential for growth in culture. Science 250, 1262-1266 (1990).

69. K. Goldsmith, W. Chen, D. Johnson, R. Hendricks, Infected Cell Protein (ICP)47 Enhances Herpes SimplexVirus Neurovirulence by Blocking the CD8? T Cell Response. J. Exp. Med. 187, 341-348 (1998).

70. A. I. Marcy, D. R. Yager, D. M. Coen, Isolation and characterization of herpes simplex virus mutants containing engineered mutations at the DNA polymerase locus. Journal of Virology 64, 2208 (1990).

71. K. Smith, Relationship between the envelope and the infectivity of herpes simplex virus. Exp Biol Med 115, 814-815 (1964).

72. N. A. Deluca, P. A. Schaffer, Activities of herpes simplex virus type $1(\mathrm{HSV}-1)$ ICP4 genes specifying nonsense peptides. Nucleic acids research 15, 4491-4511 (1987).

73. L. A. Samaniego, N. Wu, N. A. DeLuca, The herpes simplex virus immediateearly protein ICPO affects transcription from the viral genome and infected-cell survival in the absence of ICP4 and ICP27. J Virol 71, 4614-4625 (1997).

74. A. Buvoli, M. Buvoli, L. Leinwand, Enhanced detection of tRNA isoacceptors by combinatorial oligonucleotide hybridization. RNA 6, 912-918 (2000).

75. J. Dembowski, N. DeLuca, Temporal viral genome-protein interactions define distinct stages of productive herpesviral infection. MBio 9, e01182-01118 (2018).

76. E. Afgan et al., The galaxy platform for accessible, reproducible and collaborative biomedical analyses: 2018 update. Nucleic Acids Res 46, W537-W544 (2018).

77. H. AD, H. JM, C. PP, L. TM, tRNA Analysis of eXpression (tRAX): A tool for integrating analysis of tRNAs, tRNA-derived small RNAs, and tRNA modifications. (2020 (submitted)).

78. P. Chan, T. Lowe, GtRNAdb 2.0: an expanded database of transfer RNA genes identified in complete and draft genomes. Nucleic Acids Res 44, D184-D189 (2016).

79. P. Chan, T. Lowe, GtRNAdb: A database of transfer RNA genes detected in genomic sequence. Nucleic Acids Res 37, D93-D97 (2009).

80. T. Lowe, P. Chan, tRNAscan-SE On-line: Search and Contextual Analysis of Transfer RNA Genes. Nucleic Acids Res 44, W54-57 (2016).

81. D. Kim, B. Langmead, S. Salzberg, HISAT: a fast spliced aligner with low memory requirements. Nat. Methods 12, 357-360 (2015).

82. Y. Liao, G. Smyth, W. Shi, featureCounts: an efficient general purpose program for assigning sequence reads to genomic features. Bioinformatics 30, 923-930 (2014). 
1100 83. B. Langmead, S. Salzberg, Fast gapped-read alignment with Bowtie 2. Nat. $1101 \quad$ Methods 9, 357-359 (2012).

1102 84. F. Ramírez et al., deepTools2: a next generation web server for deep1103 sequencing data analysis. Nucleic Acids Res 44, W160-W165 (2016). 


\begin{tabular}{|c|c|c|c|c|c|c|c|}
\hline 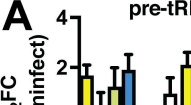 & 1 & 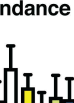 & . & $\begin{array}{l}\mathrm{n} 12 \\
\text { 5dl1.2 }\end{array}$ & 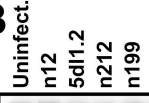 & 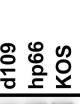 & \\
\hline 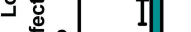 & & & & & $-2-=$ & $y=$ & IIle \\
\hline & & & & hp66 & ---- & --- & Arg \\
\hline & & & & KOS & $----\infty$ & $-=-$ & Tyr \\
\hline Ile & Arg & Ty & & & & & \\
\hline Virus & n12 & 5dl1.2 & n212 & n199 & d109 & hp66 & KOS \\
\hline Genotype & $\triangle \mathrm{ICP} 4$ & $\triangle \mathrm{ICP} 27$ & $\triangle \mathrm{ICP} 0$ & $\triangle \mathrm{ICP} 22$ & $\Delta \mathrm{ICP} 0 / 4 / 22 / 27 / 47$ & $\Delta$ UL30 & WT \\
\hline Viral nuclear entry & + & + & + & + & + & + & + \\
\hline IE transcription & ++++ & +++ & +++ & ++ & - & ++ & +++ \\
\hline E transcription & - & + & ++ & ++ & - & ++ & +++ \\
\hline$L$ transcription & - & + & ++ & ++ & - & + & +++ \\
\hline Genome replication & - & + & +++ & +++ & - & - & +++ \\
\hline Host Transcription & - & - & $\cdots$ & $\cdots$ & + & + & $\cdots$ \\
\hline Immune Response & - & - & - & - & +++ & - & - \\
\hline tRNA Upregulation & +++ & ++ & +++ & +++ & - & - & +++ \\
\hline
\end{tabular}

\section{D 总}

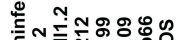

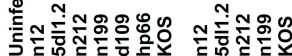

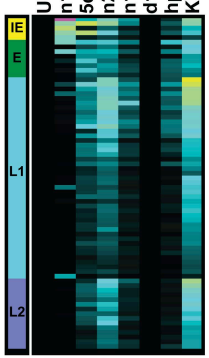

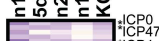

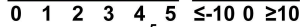
Norm MR $\left(10^{5}\right) \quad \log _{2} \mathrm{FC}$
트

Transcript-Abundance
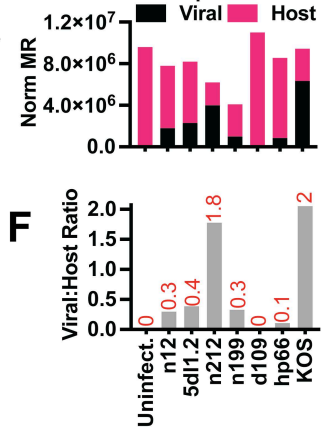


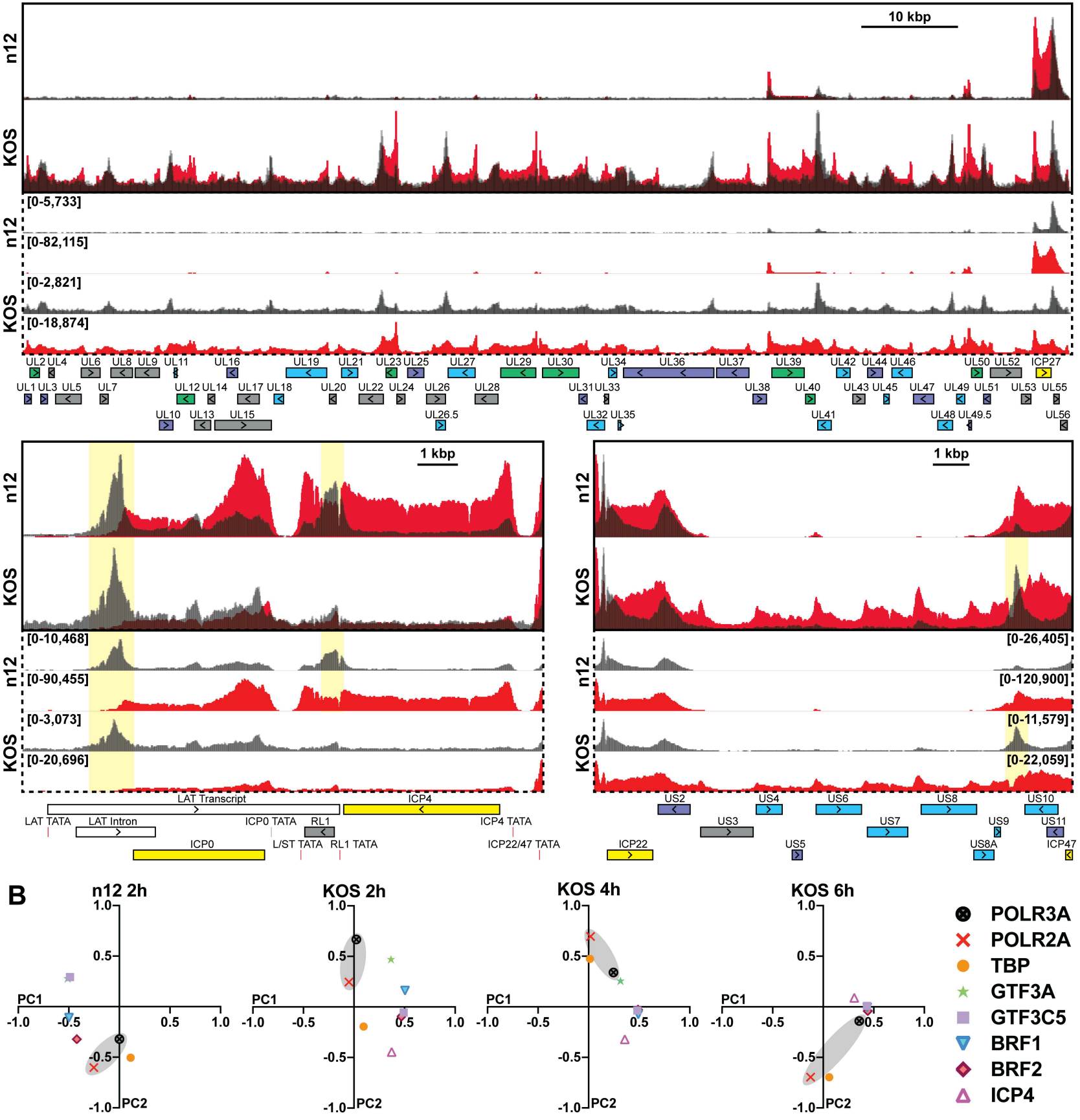


Pol II independent binding

Pol II coincident binding

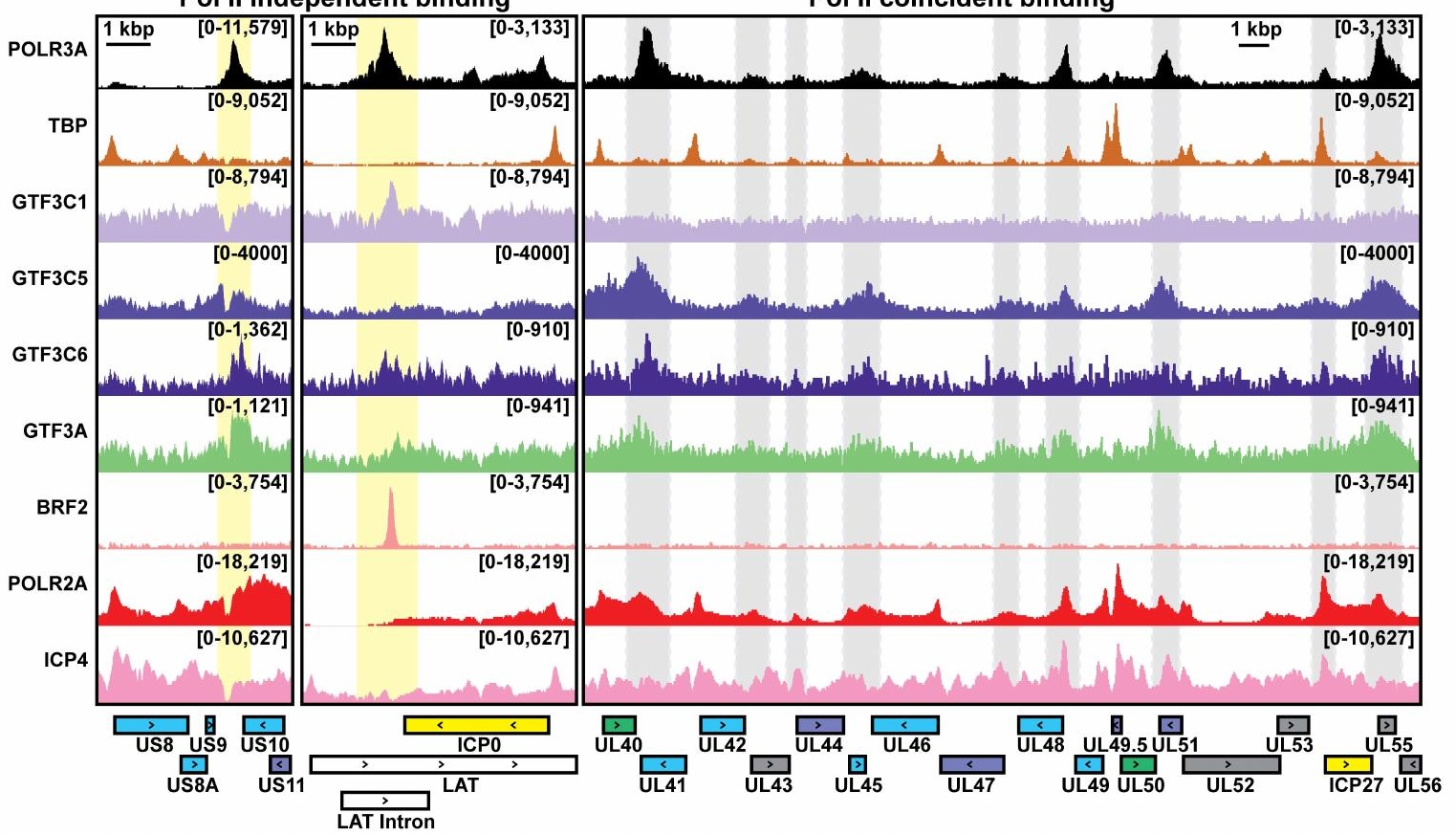



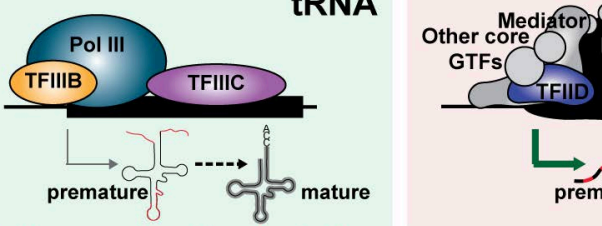

mRNA

"host response to rapid synthesis of
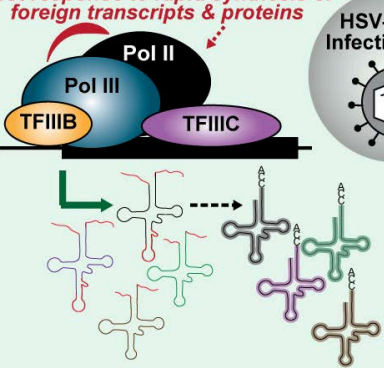

HSV-1 Lytic
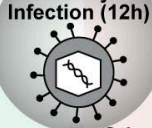

host gene

host transcriptional shut off Pol II

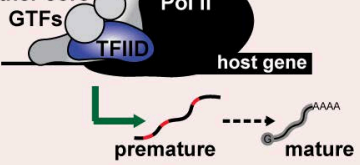

